\title{
RIM Controls Homeostatic Plasticity through Modulation of the Readily-Releasable Vesicle Pool
}

\author{
Martin Müller, ${ }^{1}$ Karen Suk Yin Liu, ${ }^{2,3}$ Stephan J. Sigrist, ${ }^{2,3}$ and Graeme W. Davis ${ }^{1}$ \\ ${ }^{1}$ Department of Biochemistry and Biophysics, University of California, San Francisco, San Francisco, California 94158-0822, ${ }^{2}$ Genetics, Institute for Biology, \\ Free University of Berlin, 14195 Berlin, Germany, and ${ }^{3}$ NeuroCure Cluster of Excellence, Charité Berlin, 10117 Berlin, Germany
}

Rab3 interacting molecules (RIMs) are evolutionarily conserved scaffolding proteins that are located at presynaptic active zones. In the mammalian nervous system, RIMs have two major activities that contribute to the fidelity of baseline synaptic transmission: they concentrate calcium channels at the active zone and facilitate synaptic vesicle docking/priming. Here we confirm that RIM has an evolutionarily conserved function at the Drosophila neuromuscular junction and then define a novel role for RIM during homeostatic synaptic plasticity. We show that loss of RIM disrupts baseline vesicle release, diminishes presynaptic calcium influx, and diminishes the size of the readily-releasable pool (RRP) of synaptic vesicles, consistent with known activities of RIM. However, loss of RIM also completely blocks the homeostatic enhancement of presynaptic neurotransmitter release that normally occurs after inhibition of postsynaptic glutamate receptors, a process termed synaptic homeostasis. It is established that synaptic homeostasis requires enhanced presynaptic calcium influx as a mechanism to potentiate vesicle release. However, despite a defect in baseline calcium influx in rim mutants, the homeostatic modulation of calcium influx proceeds normally. Synaptic homeostasis is also correlated with an increase in the size of the RRP of synaptic vesicles, although the mechanism remains unknown. Here we demonstrate that the homeostatic modulation of the RRP is blocked in the rim mutant background. Therefore, RIM-dependent modulation of the RRP is a required step during homeostatic plasticity. By extension, homeostatic plasticity appears to require two genetically separable processes, the enhancement of presynaptic calcium influx and a RIM-dependent modulation of the RRP.

\section{Introduction}

Throughout the nervous system, homeostatic signaling systems are thought to stabilize neural function through the regulation of ion channel density, neurotransmitter receptor abundance, and presynaptic neurotransmitter release (Davis, 2006; Marder and Goaillard, 2006; Bergquist et al., 2010). We have published previously a large-scale effort to identify genes involved in the homeostatic modulation of presynaptic release at the Drosophila neuromuscular junction (NMJ) (Dickman and Davis, 2009; Müller et al., 2011). In brief, inhibition of postsynaptic glutamate receptors at the Drosophila NMJ induces a retrograde signaling system that causes an increase in presynaptic neurotransmitter release. The increase in release precisely offsets the postsynaptic perturbation and restores muscle excitation in the continued presence of the perturbation, evidence of a homeostatic signaling system. The homeostatic modulation of presynaptic release is blocked by point mutations in the $\alpha 1$ subunit of the presynaptic

\footnotetext{
Received Feb. 27, 2012; revised Aug. 6, 2012; accepted Sept. 4, 2012.

Author contributions: M.M. and G.W.D. designed research; M.M. performed research; K.S.Y.L. and S.J.S. contributed unpublished reagents/analytic tools; M.M. analyzed data; M.M. and G.W.D. wrote the paper.

This work was supported by Swiss National Science Foundation Fellowship PBSKP3-123456/1 (M.M.), SFB958, TP A03 and A06, SFB 665 TP B9 (S.J.S.), and National Institutes of Health Grant NS39313 (G.W.D.). We thank the members of the Davis laboratory for comments and discussion and Cody Locke for assistance in acquisition of SIM microscopy images.

Correspondence should be addressed to Graeme W. Davis, Department of Biochemistry and Biophysics, 1550 Fourth Street, Rock Hall, Fourth Floor North, University of California, San Francisco, San Francisco, CA 94158-0822. E-mail: graeme.davis@ucsf.edu.

DOI:10.1523/JNEUROSCI.0981-12.2012

Copyright $\odot 2012$ the authors $\quad 0270-6474 / 12 / 3216574-12 \$ 15.00 / 0$
}

calcium channel $\mathrm{Ca}_{\mathrm{V}} 2.1$ (Frank et al., 2006). A recent calcium imaging study has extended these genetic data, demonstrating that the homeostatic enhancement of release requires increased calcium influx through the $\mathrm{Ca}_{\mathrm{V}} 2.1$ calcium channel (Müller and Davis, 2012). Mechanistically, it has been shown that Rab3-GAP, which acts in concert with the presynaptic vesicle-associated protein Rab3, is also necessary for homeostatic synaptic plasticity (Müller et al., 2011). Based on the involvement of both Rab3 signaling and the $\mathrm{Ca}_{\mathrm{V}} 2.1$ calcium channel, we hypothesized that the Rab3 interacting molecule (RIM), which biochemically interacts with both Rab3 and calcium channels, might be centrally involved in homeostatic plasticity.

RIMs are evolutionarily conserved scaffolding proteins that are located at presynaptic active zones (Wang et al., 1997; Koushika et al., 2001; Mittelstaedt et al., 2010). Electrophysiological analyses of mammalian synapses lacking RIM1 or RIM2 isoforms demonstrate a role for RIMs in the control of synaptic transmission (Schoch et al., 2002; Calakos et al., 2004; Schoch et al., 2006). Most recently, analysis of RIM1/2 double knock-out mice has shown that RIMs concentrate calcium channels at the presynaptic active zone (Han et al., 2011; Kaeser et al., 2011a) and facilitate synaptic vesicle docking at the presynaptic release site (Deng et al., 2011). In addition to the involvement of RIM in synaptic baseline transmission, different RIM isoforms are required for LTP and LTD at various synapses (Castillo et al., 2002, 2011).

Drosophila is predicted to encode a single rim gene (Wang and Südhof, 2003), facilitating a loss-of-function genetic analysis of rim function in Drosophila (Graf et al., 2012). Here we provide a 
genetic dissection of rim function during homeostatic regulation of transmitter release at the Drosophila NMJ. We show that rim has an evolutionarily conserved function to promote baseline presynaptic calcium influx, vesicle release, and readily-releasable pool (RRP) size. We go on to demonstrate that rim is specifically required during homeostatic synaptic plasticity by acting on the RRP and not on the homeostatic modulation of presynaptic calcium influx. Our data not only define a novel activity for RIM but also provide a novel molecular mechanism for the homeostatic control of neurotransmitter release.

\section{Materials and Methods}

Fly stocks and genetics. Drosophila stocks were maintained at $22-25^{\circ} \mathrm{C}$ on normal food. Unless otherwise noted, all fly lines were obtained from the Bloomington Drosophila Stock Center or the Exelixis Collection (Harvard Medical School). UAS-RIM RNAi animals were obtained from the Vienna Drosophila Stock Center (stock GD15273). Standard second and third chromosome balancers and genetic strategies were used for all crosses and for maintaining mutant lines. The rim $^{103}$ allele was generated by imprecise excision of parental transposon P\{EPgy2\}Rim[EY05246] (insertion position: 13,710,797), 393 bp upstream of exon 16 (see Fig. $1 A$ ). For pan-neuronal expression, we used driver $e^{2 l a v}{ }^{c 155}-$ Gal4 on the $\mathrm{X}$ chromosome (male larvae) in combination with UAS-dicer2 on the second chromosome (Dietzl et al., 2007). Unless noted, male and female larvae were used. Unless otherwise noted, the $w^{1118}$ strain was used as a wild-type (WT) control.

Electrophysiology. Sharp-electrode recordings were made from muscle 6 in abdominal segments 2 and 3 of third-instar larvae using an Axopatch 200B or a Multiclamp 700B amplifier (Molecular Devices) as described previously (Davis and Goodman, 1998). Two-electrode voltage-clamp recordings were performed with an Axoclamp 2B amplifier. The extracellular HL3 saline contained the following (in $\mathrm{mm}$ ): $70 \mathrm{NaCl}, 5 \mathrm{KCl}, 10$ $\mathrm{MgCl}_{2}, 10 \mathrm{NaHCO}_{3}, 115$ sucrose, 4.2 trehalose, 5 HEPES, and 0.4 (unless specified) $\mathrm{CaCl}_{2}$. For acute pharmacological homeostatic challenge, larvae were incubated in Philanthotoxin-433 (PhTX; 10 or $20 \mu \mathrm{m}$; SigmaAldrich) for $10 \mathrm{~min}$ (Frank et al., 2006). EGTA-AM (25 $\mu \mathrm{M}$ in HL3; Invitrogen) was applied to the dissected preparation for $10 \mathrm{~min}$. After EGTA application, the preparation was washed with HL3 for $5 \mathrm{~min}$. The average single action potential (AP)-evoked EPSP amplitude (stimulus duration, $3 \mathrm{~ms}$ ) or EPSC amplitude of each recording is based on the mean peak EPSP amplitude or EPSC amplitudes in response to 30 presynaptic stimuli unless specified. For each recording, we analyzed at least 100 miniature EPSPs (mEPSPs) to obtain a mean mEPSP amplitude value. Quantal content was estimated for each recording by calculating the ratio of EPSP amplitude/average mEPSP amplitude and then averaging recordings across all NMJs for a given genotype. EPSC data were analyzed in the same way.

The apparent size of the RRP was probed by the method of cumulative EPSC amplitudes (Schneggenburger et al., 1999), which was recently applied to the Drosophila NMJ (Hallermann et al., 2010; Miśkiewicz et al., 2011; Weyhersmüller et al., 2011). Muscles were clamped to $-65 \mathrm{mV}$, and EPSC amplitudes during a stimulus train $(60 \mathrm{~Hz}, 30$ stimuli) were calculated as the difference between peak and baseline before stimulus onset of a given EPSC. The number of release-ready vesicles was obtained by back-extrapolating a line fit to the linear phase of the cumulative EPSC plot (the last $200 \mathrm{~ms}$ of a train) to time 0 (see Fig. $7 A, B$, bottom). The number of release-ready vesicles is then obtained by dividing the cumulative EPSC amplitude at time 0 by the mean mEPSC amplitude recorded in the same cell (see Fig. 7C, right). Because of initial facilitation/delayed depression of EPSC amplitudes during trains under conditions of reduced release probability $\left(0.4 \mathrm{~mm}\left[\mathrm{Ca}^{2+}\right]_{\mathrm{e}}\right)$, the RRP at low $\left[\mathrm{Ca}^{2+}\right]_{\mathrm{e}}$ was assessed with longer trains (100 stimuli), and the RRP size estimate was based on a later linear phase of the cumulative EPSC data $(>1.2 \mathrm{~s}$; see Fig. $7 D)$. It is worth noting that the resulting RRP estimate at $0.4 \mathrm{~mm}\left[\mathrm{Ca}^{2+}\right]_{\mathrm{e}}$ may overestimate the total RRP as a result of "recovery from depression" (Schneggenburger et al., 1999; Weyhersmüller et al., 2011).

For fluctuation analysis (see Fig. 8), the mean EPSC amplitude ( $I$ ) and the EPSC amplitude variance of each synapse at each extracellular cal- cium concentration $\left(\left[\mathrm{Ca}^{2+}\right]_{e} ; 0.3,1\right.$, and $\left.3 \mathrm{~mm} ;\left[\mathrm{Mg}^{2+}\right]_{\mathrm{e}}, 10 \mathrm{~mm}\right)$ was based on 40-150 consecutive EPSCs (interstimulus interval, 5 s). EPSC amplitude variance was calculated according to previous reports (Meyer et al., 2001; Scheuss and Neher, 2001; Scheuss et al., 2002), and the quantal parameters $N$ and $q$ were obtained by fitting the EPSC variancemean data of each synapse with a parabola $\left[\operatorname{Var}(I)=I^{2} / N+q I\right]$ that was constrained to pass through the origin. $N$ and $q$ values were then averaged across cells. The mean coefficients of variation of mEPSC amplitudes of all groups were similar (data not shown), and values for $q$ and $N$ were not corrected for variability in mEPSC amplitude distributions (Brown et al., 1976; Silver et al., 1998; Scheuss and Neher, 2001) or latency fluctuations (“jitter") of individual quantal events (Taschenberger et al., 2005; Weyhersmüller et al., 2011).

$\mathrm{Ca}^{2+}$ imaging. $\mathrm{Ca}^{2+}$ imaging experiments were done as described by Müller and Davis (2012). Third-instar larvae were dissected and incubated in ice-cold, $\mathrm{Ca}^{2+}$-free HL3 containing $5 \mathrm{~mm}$ Oregon-Green 488 BAPTA-1 (OGB-1) (hexapotassium salt; Invitrogen) and $1 \mathrm{~mm}$ Alexa Fluor 568 (Invitrogen). After incubation for $10 \mathrm{~min}$, the preparation was washed with ice-cold HL3 for 10-15 min. Single action-potential evoked spatially averaged $\mathrm{Ca}^{2+}$ transients were measured in type- $1 \mathrm{~b}$ boutons synapsing onto muscle $6 / 7$ of abdominal segments A2/A3 at an $\left[\mathrm{Ca}^{2+}\right]_{\mathrm{e}}$ of $1 \mathrm{~mm}$ using a confocal laser-scanning system (Ultima; Prairie Technologies $)$ at room temperature. Excitation light $(488 \mathrm{~nm})$ from an aircooled krypton-argon laser was focused onto the specimen using a $60 \times$ objective (1.0 NA; Olympus), and emitted light was detected with a gallium arsenide phosphide-based photocathode photomultiplier tube (Hamamatsu). Line scans across single boutons were made at a frequency of $313 \mathrm{~Hz}$. Fluorescence changes were quantified as $\Delta F / F=(F(t)-$ $\left.F_{\text {baseline }}\right) /\left(F_{\text {baseline }}-F_{\text {background }}\right)$, where $F(t)$ is the fluorescence in a region of interest (ROI) containing a bouton at any given time, $F_{\text {baseline }}$ is the mean fluorescence from a $300 \mathrm{~ms}$ period preceding the stimulus, and $F_{\text {background }}$ is the background fluorescence from an adjacent ROI without any indicator-containing cellular structures. One synapse (4-12 boutons) was imaged per preparation. The average $\mathrm{Ca}^{2+}$ transient of a single bouton is based on $8-12$ line scans. Experiments in which the resting fluorescence decreased by $>15 \%$ and/or which had an $F_{\text {baseline }}>650$ a.u. were excluded from analysis. Data of experimental and control groups were collected side by side. The $\mathrm{Ca}^{2+}$ indicator was not saturated by single AP stimulation because repetitive stimulation induced an additional increase in peak $\Delta F / F(20 \mathrm{~ms}$ interstimulus interval; data not shown). The intraterminal $\mathrm{Ca}^{2+}$ indicator concentration $(\sim 50 \mu \mathrm{M})$ was roughly approximated by an in vitro calibration (Müller and Davis, 2012).

Data analyses. Electrophysiology data and $\mathrm{Ca}^{2+}$ imaging data were analyzed with custom-written routines in Igor Pro 6.22 (Wavemetrics), and spontaneous mEPSPs were analyzed with Mini Analysis 6.0.0.7 (Synaptosoft). $\mathrm{Ca}^{2+}$ imaging data was acquired with Prairie View. Deconvolution microscopy data (see Fig. $2 A, B$ ) were acquired and analyzed with Intelligent Imaging Innovations (3i) software. Structured-illumination (SIM) data (Fig. 2C-E) was acquired with ZEN software (Carl Zeiss) and analyzed with custom-written macros in NIH ImageJ/Fiji (W. S. Rasband, National Institutes of Health, Bethesda, MD; Schindelin et al., 2012) and Igor Pro. All results are reported as average \pm SEM. Statistical significance was assessed by Student's $t$ test unless otherwise specified.

Quantitative RT-PCR. Quantitative RT-PCR was performed as described by Berquist et al. (2010). Primer probes were designed and developed by Applied Biosystems. The CNS was removed from 25 third-instar larvae per sample (three samples per genotype). Total RNA was isolated from each sample using the standard Trizol protocol. A DNase digestion removed potential DNA contamination (RQ1 RNase-free DNase; Promega). RT was performed (Taqman reverse transcription reagents; Applied Bioscience) using random hexamers and $1 \mu \mathrm{g}$ of total RNA. A no-RT control was performed for each sample. Purified cDNA was used as a template in $30 \mu \mathrm{l}$ of PCR reaction (TaqMan Universal PCR Master Mix, no AmpErase UNG; Applied Biosystems). This $30 \mu \mathrm{l}$ reaction was divided into three $10 \mu \mathrm{l}$ triplicates. In addition, one $10 \mu \mathrm{l}$ no-RT reaction was used for each sample. The ABI Prism 7900 was used for all PCRs. Cycle threshold $\left(\mathrm{C}_{\mathrm{T}}\right)$ was determined by automated threshold analysis using SDS2.3 software according to the instructions of the manufacturer 
A RNA $_{i}$ GD15273

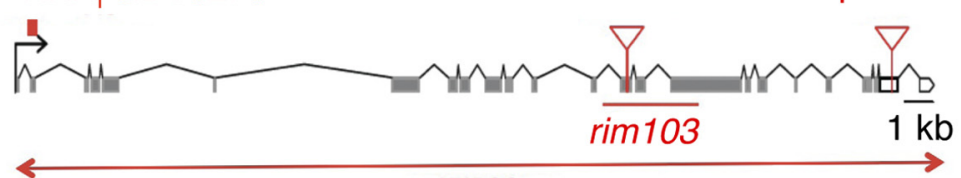

Df(3R)BSC650

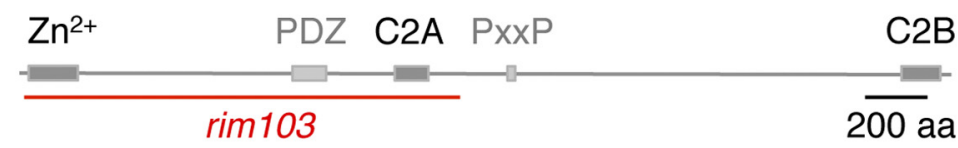

B
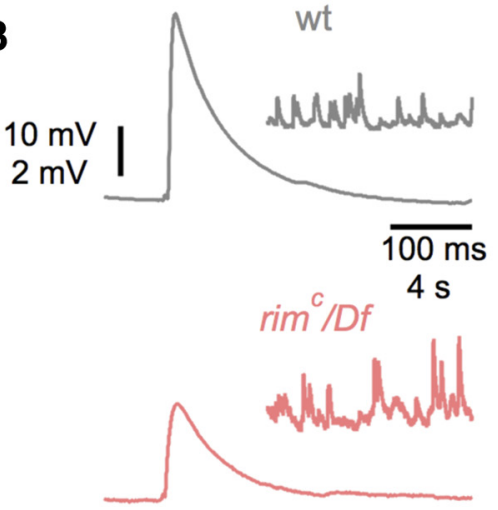

$\operatorname{rim}^{c} / D f+\operatorname{PhTX}$

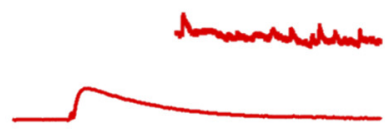

C
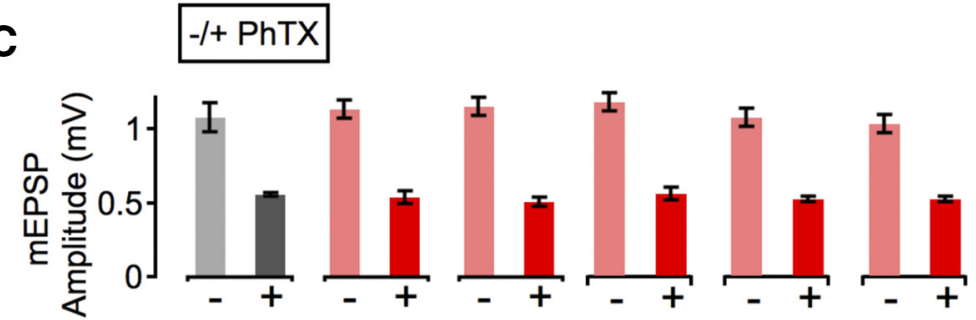

D

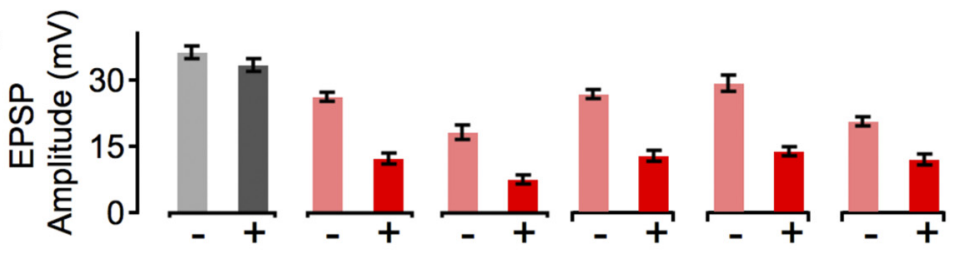

E

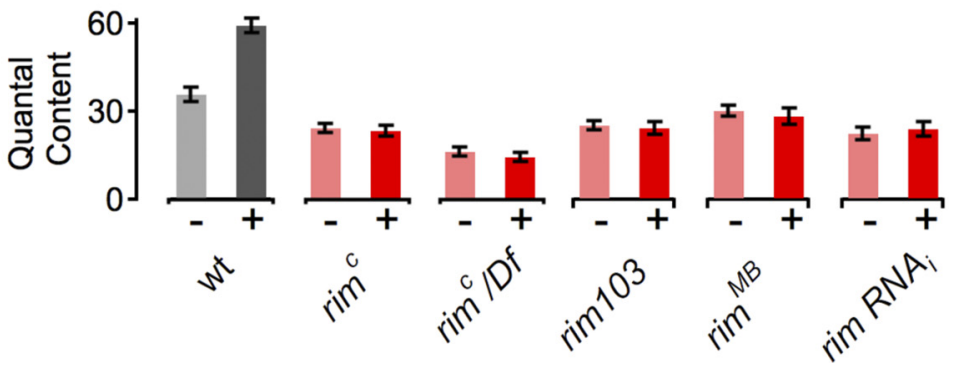

Figure 1. Identification of rim as an essential gene for synaptic homeostasis. $\boldsymbol{A}$, Top, Schematic of the rim locus indicating the approximate location of each mutation and the RNAi transgene. Coding exons are gray, and noncoding are open boxes. Bottom, Schematic of the Rim protein structure. Boxes indicate the respective locations of the zinc finger domain $\left(\mathrm{Zn}^{2+}\right)$, the central PDZ domain, the proline-rich sequence (PxxP), and the $\left(2 A\right.$ and $\left(2 B\right.$ domains. The red line shows the approximate location of the rim ${ }^{103}$ deletion. $\boldsymbol{B}$, Sample traces showing EPSP and mEPSP amplitudes for WT in the absence and presence of PhTX (gray and black data, respectively) and rim ${ }^{C}$ placed over the deficiency chromosome Df(3R)BSC650 in the absence and presence of PhTX (light red and dark red data, respectively). C, Average data for mEPSP amplitude for indicated genotypes minus PhTX (light gray/red) and plus PhTX (black, dark red). $w^{1118}(-\mathrm{PhTx}), n=12 ; w^{1118}(+\mathrm{PhTx}), n=13 ; \operatorname{rim}^{c}(-\mathrm{PhTx}), n=35 ; \operatorname{rim}^{c}(+\mathrm{PhTx}), n=22 ; \operatorname{rim}^{c} / \mathrm{D}$ $(-\mathrm{PhTx}), n=15 ; \mathrm{rim}^{\mathrm{C}} / \mathrm{Df}(+\mathrm{PhTx}), n=13 ; \operatorname{rim}^{103}(-\mathrm{PhTx}), n=20 ; \operatorname{rim}^{103}(+\mathrm{PhTx}), n=12 ; \operatorname{rim}^{M B}(-\mathrm{PhTx}), n=11 ; \operatorname{rim}^{M B}$
(Applied Biosystems). Comparative levels (between WT and mutant animals) were determined using the $\Delta \Delta \mathrm{C}_{\mathrm{T}}$ method (Applied Biosystems User Bulletin 2). To determine whether the two amplification reactions have the same PCR efficiency, $\Delta \mathrm{C}_{\mathrm{T}}\left(\mathrm{C}_{\mathrm{T}}\right.$ of experimental gene $-\mathrm{C}_{\mathrm{T}}$ of reference gene) values are determined across the serial dilutions and plotted against the $\log$ of the cDNA dilution. Briefly, the $\Delta \Delta \mathrm{C}_{\mathrm{T}}$ method is as follows. $\Delta \mathrm{C}_{\mathrm{T}}$ values are determined as explained above. Next, experimental animal (mutants) $\Delta \mathrm{C}_{\mathrm{T}}$ values were subtracted from control animal (WT) $\Delta \mathrm{C}_{\mathrm{T}}$ values to give the $\Delta \Delta \mathrm{C}_{\mathrm{T}}$. Finally, using the equation $2 \wedge\left(-\Delta \Delta \mathrm{C}_{\mathrm{T}}\right) \times 100$, the percentage expression of each gene in experimental compared with control animals was calculated. Each experimental animal sample was compared to each WT sample (Applied Biosystems User Bulletin No. 2).

Synapse morphology. Third-instar larval preparations were fixed for $2 \mathrm{~min}$ in Bouin's fixative (100\%; Sigma-Aldrich) or for $15 \mathrm{~min}$ in PFA ( $4 \%$ in PBS) and incubated overnight at $4^{\circ} \mathrm{C}$ with primary antibodies. The following primary antibodies were used at the indicated dilutions: mouse anti-Bruchpilot (Brp), 1:100 (nc82; Kittel et al., 2006); and rabbit anti-Dlg, 1:5000. Alexa Fluor-conjugated secondary antibodies and Cy3-conjugated anti-HRP were used at 1:200 and 1:800, respectively (Jackson ImmunoResearch; Invitrogen), and applied for $2 \mathrm{~h}$ at room temperature. Larval preparations were mounted in Vectashield (Vector Laboratories). An Axiovert 200 inverted microscope (Carl Zeiss), a $100 \times$ (1.4 NA) Plan Apochromat objective (Carl Zeiss) and a cooled CCD camera (CoolSNAP HQ; Roper Scientific) were used for deconvolution microscopy, and data were analyzed as described previously (Pielage et al., 2008) (see Fig. 2A,B).

For SIM imaging, we used an ELYRA PS.1 system (Carl Zeiss) with an inverted LSM-710 microscope, a $63 \times(1.4 \mathrm{NA})$ Plan-Apochromat objective (Carl Zeiss), and an Andor iXon 885 EMCCD camera. Lateral resolution was $\sim 110$ $\mathrm{nm}$, and axial resolution was $\sim 300 \mathrm{~nm}$. $Z$-stacks of whole NMJs at muscle 4 were taken with oversampling in $x y(40 \times 40 \mathrm{~nm}$ pixel size) and $z$ (110 nm step size). Individual Brp puncta were identified with a threshold-based mask applied to the maximum projection of a

(+PhTx), $n=14 ;$ rim RNAi (-PhTx), $n=21$; rim RNAi $(+\mathrm{PhTx}), n=14$. PhTX application significantly reduced $\mathrm{mEPSP}$ amplitude compared with the respective baseline in all groups (all $p<0.05$ ). D, Average EPSP amplitude as in C. EPSP amplitudes of PhTX and control group were similar in WT ( $p=$ 0.17 ) but differed significantly in $\mathrm{rim}^{c}, \mathrm{rim}^{C} / \mathrm{Df}, \mathrm{rim}^{103}, \mathrm{rim}^{\mathrm{MB}}$, and after neural expression of UAS--rim RNAi (rim RNAi; see Results; all $p<0.001)$. $E$, Average data for quantal content, as in $C$. Although PhTX treatment induced an increase in quantal content in WT $(p<0.01)$, there was no significant difference in quantal content during $\mathrm{PhTX}$ application in all rim mutants (all $p>0.05$ ), indicating a block in homeostatic plasticity. All data represent mean \pm SEM. For averages corresponding to the data presented in this figure, see Table 1. 
Table 1. Summary of raw data

\begin{tabular}{|c|c|c|c|c|c|c|c|c|c|}
\hline Figure & Genotype & Condition $(-/+\mathrm{PhTX})$ & $\begin{array}{l}\text { mEPSP } \\
\text { amplitude (mV) }\end{array}$ & $\begin{array}{l}\text { EPSP } \\
\text { amplitude (mV) }\end{array}$ & $\begin{array}{l}\text { Quantal } \\
\text { content }\end{array}$ & $\begin{array}{l}\text { mEPSC } \\
\text { amplitude (nA) }\end{array}$ & $\begin{array}{l}\text { Cum. EPSC } \\
\text { amplitude (nA) }\end{array}$ & $\begin{array}{l}\text { RRP } \\
\text { (vesicles) }\end{array}$ & $n$ \\
\hline \multirow[t]{12}{*}{1} & $w^{1118}$ & - & $1.08 \pm 0.1$ & $36.3 \pm 1.5$ & $36.1 \pm 2.6$ & & & & 12 \\
\hline & $w^{1118}$ & + & $0.56 \pm 0.01$ & $33.4 \pm 1.5$ & $59.6 \pm 2.4$ & & & & 13 \\
\hline & $\mathrm{rim}^{\mathrm{c}}$ & - & $1.14 \pm 0.06$ & $26.2 \pm 1.1$ & $24.5 \pm 1.4$ & & & & 35 \\
\hline & $\mathrm{rim}^{c}$ & + & $0.54 \pm 0.04$ & $12.4 \pm 1.3$ & $23.5 \pm 2.0$ & & & & 22 \\
\hline & $\mathrm{rim}^{C} / D f[B S C 650]$ & - & $1.16 \pm 0.06$ & $18.3 \pm 1.7$ & $16.5 \pm 1.7$ & & & & 15 \\
\hline & $\mathrm{rim}^{c} / D f[B S C 650]$ & + & $0.51 \pm 0.03$ & $7.6 \pm 1.0$ & $14.5 \pm 1.5$ & & & & 13 \\
\hline & $\mathrm{rim}^{103}$ & - & $1.14 \pm 0.06$ & $26.2 \pm 1.1$ & $24.5 \pm 1.4$ & & & & 20 \\
\hline & $\operatorname{rim}^{103}$ & + & $0.54 \pm 0.04$ & $12.4 \pm 1.3$ & $23.5 \pm 2.0$ & & & & 12 \\
\hline & $\mathrm{rim}^{\mathrm{MB}}$ & - & $1.04 \pm 0.06$ & $28.7 \pm 1.8$ & $29.5 \pm 1.9$ & & & & 11 \\
\hline & $\mathrm{rim}^{\mathrm{MB}}$ & + & $0.5 \pm 0.02$ & $13.3 \pm 1.0$ & $27.6 \pm 2.7$ & & & & 14 \\
\hline & c155>rim RNAi & - & $1.0 \pm 0.06$ & $20.1 \pm 1.1$ & $21.7 \pm 2.1$ & & & & 21 \\
\hline & c155>rim RNAi & + & $0.5 \pm 0.02$ & $11.6 \pm 1.2$ & $23.4 \pm 2.5$ & & & & 14 \\
\hline \multirow[t]{4}{*}{6} & $w^{1118}$ & $-(1 C a)$ & $0.91 \pm 0.15$ & $50.1 \pm 1.4$ & $177 \pm 20$ & & & & 10 \\
\hline & $w^{1118}$ & $+(1 \mathrm{Ca})$ & $0.50 \pm 0.03$ & $54.9 \pm 2.5$ & $313 \pm 22$ & & & & 8 \\
\hline & $\operatorname{rim}^{103}$ & $-(1 \mathrm{Ca})$ & $0.95 \pm 0.06$ & $40.8 \pm 1.8$ & $97 \pm 8$ & & & & 14 \\
\hline & $\operatorname{rim}^{103}$ & $+(1 \mathrm{Ca})$ & $0.51 \pm 0.02$ & $33.3 \pm 0.9$ & $124 \pm 10$ & & & & 13 \\
\hline \multirow[t]{8}{*}{8} & $w^{1118}$ & - & $1.04 \pm 0.05$ & $36.3 \pm 1.3$ & $35 \pm 2$ & & & & 10 \\
\hline & $w^{1118}$ & + & $0.52 \pm 0.02$ & $37.6 \pm 3.9$ & $73 \pm 11$ & & & & 11 \\
\hline & rab3-GAP/+ & - & $1.08 \pm 0.03$ & $31.9 \pm 1.4$ & $30 \pm 2$ & & & & 13 \\
\hline & $\mathrm{rab3}-\mathrm{GAP} /+$ & + & $0.55 \pm 0.02$ & $29.2 \pm 1.8$ & $53 \pm 3$ & & & & 15 \\
\hline & $\mathrm{rim}^{M B} /+$ & - & $1.02 \pm 0.06$ & $29.3 \pm 2.5$ & $29 \pm 2$ & & & & 12 \\
\hline & $\operatorname{rim}^{M B} /+$ & + & $0.53 \pm 0.01$ & $27.6 \pm 1.7$ & $52 \pm 3$ & & & & 12 \\
\hline & $\mathrm{rim}^{M B} / \mathrm{rab3}-\mathrm{GAP}$ & - & $1.06 \pm 0.07$ & $27.3 \pm 2.3$ & $27 \pm 2$ & & & & 18 \\
\hline & $\mathrm{rim}^{M B} / \mathrm{rab3}-\mathrm{GAP}$ & + & $0.51 \pm 0.02$ & $17.2 \pm 1.9$ & $36 \pm 4$ & & & & 20 \\
\hline \multirow[t]{8}{*}{7} & $w^{1118}$ & $-(3 \mathrm{Ca})$ & & & & $0.82 \pm 0.13$ & $502 \pm 66$ & $740 \pm 98$ & 6 \\
\hline & $w^{1118}$ & $+(3 \mathrm{Ca})$ & & & & $0.53 \pm 0.03$ & $450 \pm 51$ & $1160 \pm 132$ & 5 \\
\hline & $\mathrm{rim}^{103}$ & $-(3 \mathrm{Ca})$ & & & & $0.90 \pm 0.09$ & $391 \pm 66$ & $436 \pm 74$ & 6 \\
\hline & $\operatorname{rim}^{103}$ & $+(3 \mathrm{Ca})$ & & & & $0.59 \pm 0.05$ & $215 \pm 28$ & $361 \pm 48$ & 5 \\
\hline & $w^{1118}$ & - & & & & $0.87 \pm 0.06$ & $534 \pm 103$ & $680 \pm 118$ & 13 \\
\hline & $w^{1118}$ & + & & & & $0.64 \pm 0.02$ & $651 \pm 77$ & $651 \pm 77$ & 13 \\
\hline & $\operatorname{rim}^{103}$ & - & & & & $0.89 \pm 0.07$ & $303 \pm 52$ & $1145 \pm 114$ & 13 \\
\hline & $\operatorname{rim}^{103}$ & + & & & & $0.63 \pm 0.03$ & $195 \pm 15$ & $324 \pm 61$ & 13 \\
\hline
\end{tabular}

Data are shown as mean \pm SEM, and $n$ refers to the number of synapses. All data are average peak amplitude values and were obtained at an [Ca $\left.{ }^{2+}\right]_{\mathrm{e}}$ of $0.4 \mathrm{~mm}$ unless otherwise noted (Figs. 6, 7). Quantal content values of data in Figure 6 were corrected for nonlinear summation (Martin, 1955). Cumulative EPSC (Cum. EPSC) and RRP at the two different $\left[\mathrm{Ca}^{2+}\right]_{\mathrm{e}}$ in Figure 7 are based on different linear fits (see Materials and Methods). Data from experimental and control groups were collected side by side, and all recordings were done at room temperature. For additional details, see Materials and Methods.

Z-stack (Fouquet et al., 2009), and confluent puncta were removed manually. A fluorescence intensity line profile ( $1.2 \mu \mathrm{m}$ long, 1 pixel wide) was obtained along the major and minor axis of a bounding ellipse that was fitted to each punctum. Diameter analysis was restricted to Brp puncta with a planar orientation with respect to the focal plane. These puncta were detected by a local minimum around the centroid of the ellipse in both line profiles. The maximum "peak-to-peak diameter" of a Brp punctum was calculated as the distance between the peaks of the line profile along the major axis of the ellipse. The "diameter at halfmaximum" was computed as the maximum distance between two points at $50 \%$ of the peak of the same profile (see Fig. $2 E$ ).

\section{Results \\ RIM is required for homeostatic modulation of neurotransmitter release}

A homeostatic potentiation of synaptic transmission can be induced by application of sub-blocking concentrations of PhTX $(4-20 \mu \mathrm{M})$ to the Drosophila NMJ (Frank et al., 2006). In WT animals, application of PhTX to the NMJ causes a significant decrease in the amplitude of spontaneous mEPSPs. After several minutes, a compensatory increase in AP-evoked presynaptic vesicle release is observed that precisely offsets the decrease in mEPSP amplitude and restores EPSP amplitudes to baseline levels seen before the application of PhTX. This occurs in the continued presence of PhTX-dependent receptor inhibition and, therefore, is referred to as a homeostatic process that increases presynaptic release to offset the inhibition of postsynaptic glutamate receptors. In previous studies, this is referred to as "synaptic homeostasis," a term that will be used from here forward.

We are seeking to define the molecular mechanisms of synaptic homeostasis. In an ongoing forward genetic screen (Dickman et al., 2009; Müller et al., 2011), we identified a transposon insertion in the rim gene $\left(\right.$ rim $^{C 165}$, henceforth called rim $^{C}$ ) that blocks PhTX-induced homeostatic plasticity. We subsequently acquired two additional rim alleles. One allele is a transposon insertion (rim $^{M B 07541}$, henceforth called $\mathrm{rim}^{\mathrm{MB}}$; Fig. $1 \mathrm{~A}$, top), which resides within a coding exon and is predicted to be a strong loss-offunction mutation. The second allele is a small internal deletion $\left(\mathrm{rim}^{103}\right)$ that removes the majority of three common exons in the rim gene, including the conserved zinc finger domain, the PDZ domain, and the C2A domain (Fig. $1 A$, bottom). The rim $^{103}$ allele is also predicted to be a strong loss-of-function allele. We find that homeostatic plasticity is completely blocked in $\mathrm{rim}^{M B}$, rim $^{103}$, and when the rim $^{C}$ is placed in trans to a deficiency that uncovers the rim locus (Fig. $1 E$ ). In particular, note that the average EPSP amplitudes in all rim alleles are significantly reduced in the presence of PhTX compared with baseline EPSP amplitudes recorded for each genotype in the absence of PhTX $(p<$ 
0.001; Fig. $1 D$, Table 1). These data demonstrate that RIM is required for homeostatic modulation of presynaptic release that normally restores EPSP amplitudes to baseline values.

We next sought to knockdown rim expression selectively in the nervous system as an independent test for the requirement of rim during synaptic homeostasis. UAS-rim RNAi was acquired from the Vienna RNAi collection (Dietzl et al., 2007), and expression was driven in neurons using the neural-specific galactosidase-4 (GAL4) driver elav-GAL4 in combination with $U A S$-dicer2 to increase RNAi efficiency (Dietzl et al., 2007). Expression of UAS-dicer 2 alone does not alter synaptic homeostasis (Dickman and Davis, 2009). However, neuronal expression of UAS-rim RNAi blocks the rapid induction of synaptic homeostasis (Fig. $1 E)$. Application of PhTX causes a significant decrease in mEPSP amplitude with respect to baseline ( $p<0.001$; Fig. $1 C$ ) without a corresponding increase in quantal content $(p=0.59$; Fig. $1 E)$. The consequence is that EPSP amplitudes remain reduced in the continued presence of PhTX $(p<0.001$; Fig. 1D). Quantification of rim RNA levels by quantitative RT-PCR demonstrates a significant knockdown of gene expression ( $64 \pm 6 \%$ compared with elav-GAL4 driver control). We suspect that this quantitative RT-PCR assay underestimates the full effect of the UAS-rim RNAi in motoneurons because the effects of rim RNAi on baseline vesicle release are nearly as large as those observed in a molecularly defined deletion that removes part of the rim coding sequence $\left(\mathrm{rim}^{103}\right)$. Together, with the complete block of synaptic homeostasis after genetic disruption of the rim locus, we conclude that neuronal RIM is necessary for synaptic homeostasis at the Drosophila NMJ.

Interestingly, there is no correlation between the effects of our mutations on baseline release and the disruption of synaptic homeostasis. Our strongest rim alleles show a strong defect in baseline transmission, providing evidence that rim has an evolutionarily conserved function to establish normal baseline synaptic transmission in Drosophila. This defect is similar in magnitude to that observed in a double knock-out mouse of RIM1 and RIM2 (Han et al., 2011; Kaeser et al., 2011a). However, at least one rim allele that we have tested $\left(\mathrm{rim}^{\mathrm{MB}}\right)$ reveals only a $20 \%$ decrease in evoked release, yet synaptic homeostasis remains completely blocked. One possibility is that homeo-
A
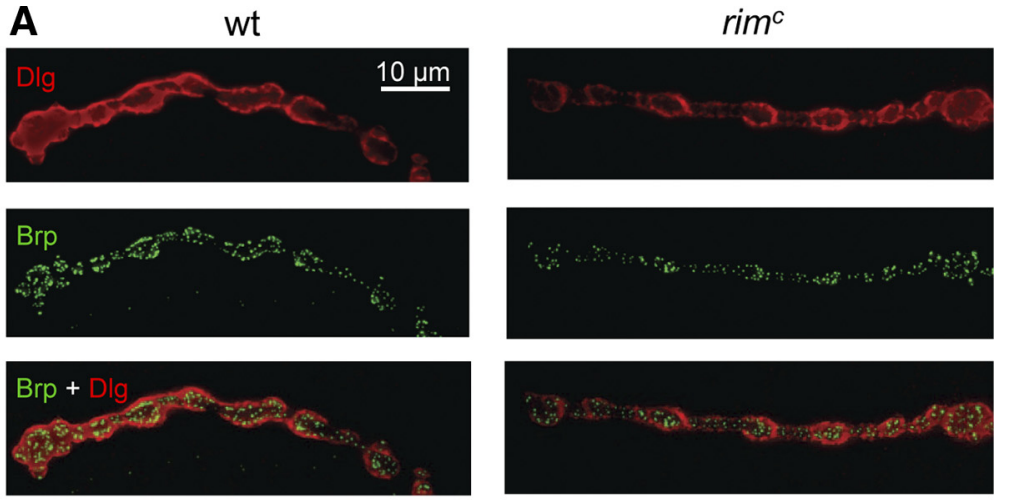

B
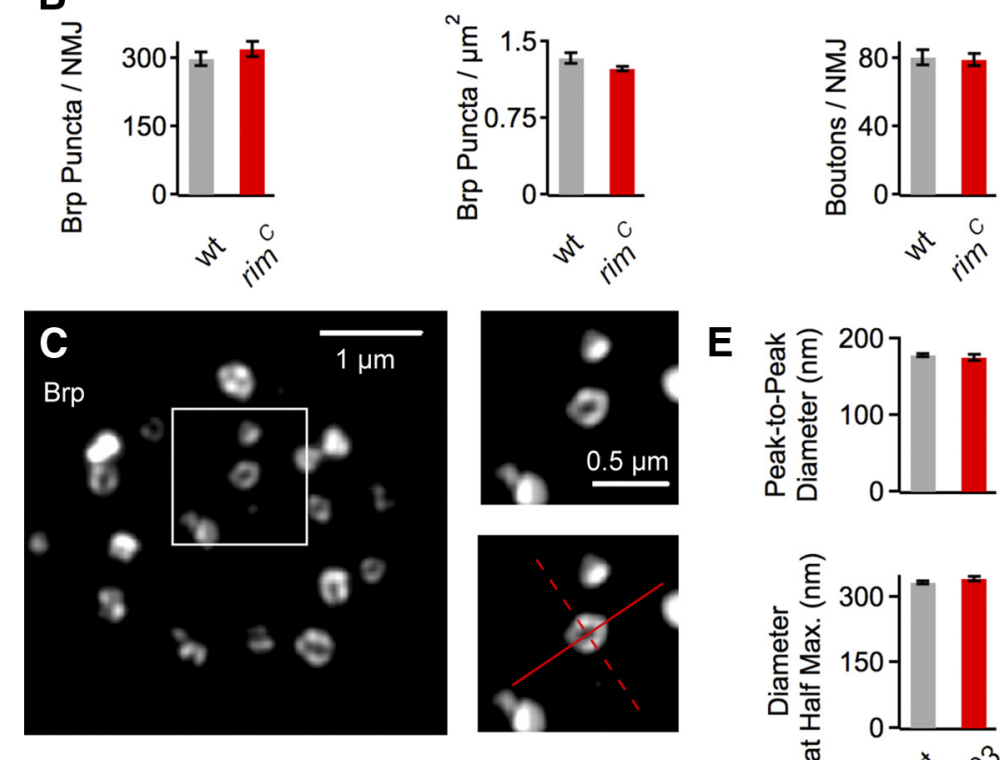

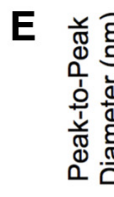

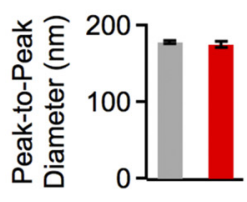

D
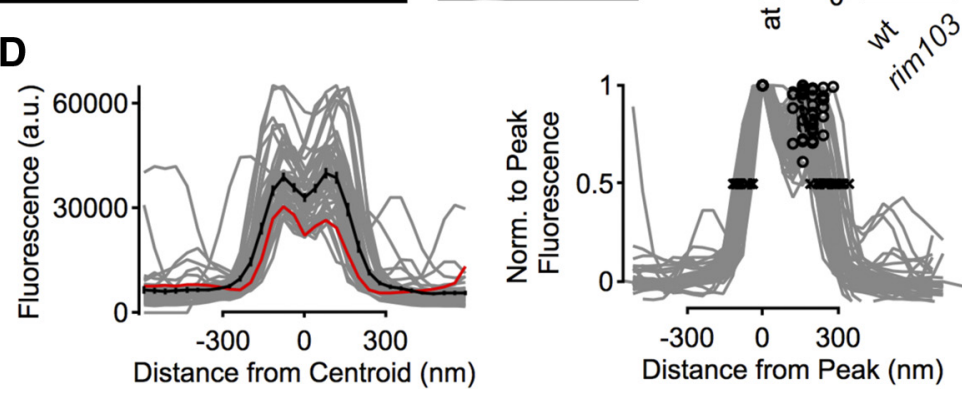

Figure 2. rim mutations do not alter synapse morphology. A, WT NMJ at muscle 4 (left) costained with the postsynaptic marker Dlg (top, red) and the presynaptic active zone marker Brp (middle, green). Merge at bottom. The rim $^{C}$ mutant synapse stained as in $\boldsymbol{A}$ is shown at right. $\boldsymbol{B}$, Quantification of Brp puncta per NMJ at muscle 4 (left), Brp puncta per square micrometer of NMJ (delineated by Dlg staining) (middle), and bouton number per NMJ at muscle 6/7. $w^{1118}, n=20$; rim $n=23$. There are no statistically significant differences in these three measures of synapse development and active zone number between the genotypes $(p>0.05)$. C, Example SIM images of active zones of a single bouton (left) and an individual active zone at increased scale (right) of a WT NMJ stained with anti-Brp. The red lines indicate the locations of the line profiles used for analysis (see Materials and Methods). Images were smoothed with (bicubic) interpolation for visualization purposes. D, Left, Fluorescence intensity line profiles through all planar Brp puncta (see Materials and Methods) of a WT NMJ (same NMJ as in C). Individual line profiles and the average line profile are shown in gray and black, respectively. The red trace is the profile of the solid red line shown in C. Right, All profiles were normalized to the respective peak fluorescence. The maximum peak-to-peak diameter of a profile was calculated as the distance between the two peaks of the intensity profile (black circles). The maximum diameter at half-maximum is the distance between two points at $50 \%$ of the peak fluorescence (black crosses). $\boldsymbol{E}$, Average maximum peak-to-peak diameter (top) and diameter at half-maximum (bottom) of Brp puncta of WT ( $n=346,9$ synapses) and $\operatorname{rim}^{103}$ ( $n=216,9$ synapses). There was no apparent change in peak-to-peak diameter $(p=0.15)$ and a slight increase in the diameter at half-maximum $(p=0.05)$ of rim $^{103} \operatorname{Brp~profiles}$ with respect to control. Together, this indicates that the organization of individual Brp puncta is essentially unaffected by rim. 

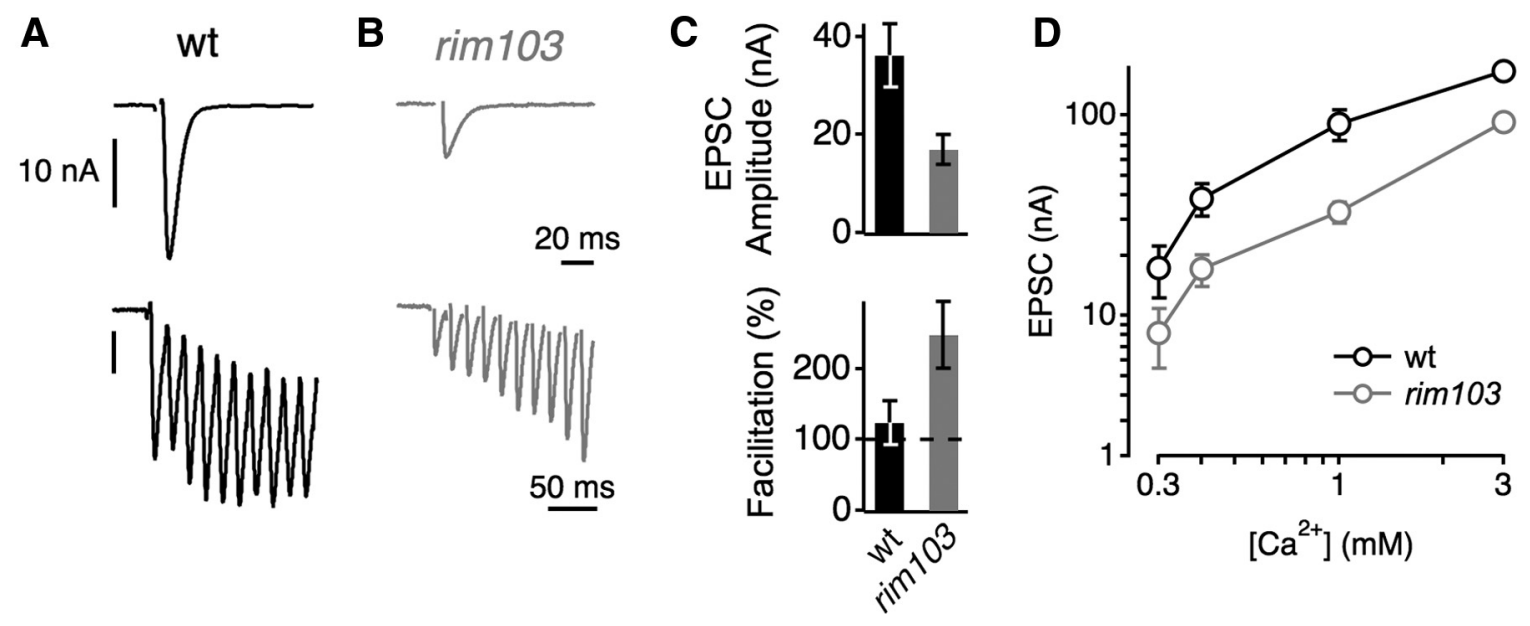

Figure 3. Evidence for decreased release probability in rim mutants. A, Representative single AP-evoked EPSC (top) and EPSCs during a $60 \mathrm{~Hz}$ train (bottom) in WT. $B$, Example EPSCs evoked by a single AP (top) and by a $60 \mathrm{~Hz}$ train (bottom) in a rim ${ }^{103}$ mutant. C, Average peak EPSC amplitude (top) and average facilitation during the stimulus train (EPSC 10/EPSC 1, bottom) in WT (black, $n=9$ ) and rim $^{103}$ (gray, $\left.n=13\right)$. The decreased EPSC amplitude ( $\boldsymbol{C}$, top) and the increased facilitation in rim ${ }^{103}$ mutants $(\boldsymbol{C}$, bottom) indicate a decrease in release probability with respect to control. $D$, Mean EPSC amplitude as a function of $\left[\mathrm{Ca}^{2+}\right]_{\mathrm{e}}$ in WT (black, $n=4-8$ per $\left[\mathrm{Ca}^{2+}\right]_{\mathrm{e}}$ ) and rim ${ }^{103}$ mutants (gray, $n=8-17$ per $\left.\left[\mathrm{Ca}^{2+}\right]_{\mathrm{e}}\right)$. The EPSC amplitudes of rim mutants were significantly smaller at all $\mathrm{Ca}^{2+}$ concentration tested $(p<0.05)$. Stimulus artifacts were removed for clarity.

static plasticity is particularly sensitive to the levels of RIM or to specific RIM isoforms. At least one RIM isoform $\left(\right.$ rim $\left.^{103}\right)$ lacks the exon in which the rim $^{M B}$ transposon resides. Regardless, because the blockade of synaptic homeostasis is not correlated with the extent of impaired baseline transmission, it suggests that the two activities of RIM may be partially separable.

\section{Normal morphology and active zone organization of rim mutant synapses}

It was demonstrated recently that loss of Rab3 causes a dramatic change in the appearance of the Drosophila NMJ, including a decrease in total active zone number and an increase in active zone size (Graf et al., 2009). Here we demonstrate that the mutations in rim that block synaptic homeostasis do not alter synaptic growth or morphology at the NMJ. The total number and density of Brp puncta (Fig. 2B), a direct reporter of individual active zones (Kittel et al., 2006), and the number of synaptic boutons (Fig. 2B, right) are unchanged compared with WT. There were also no major changes in the organization of individual active zones between WT and rim synapses as assayed by highresolution SIM microscopy (Fig. 2C-E). The average maximum diameter of "planar" Brp rings was similar between rim ${ }^{103} \mathrm{mu}$ tants and WT when quantified as the distance between the two peaks of a fluorescence intensity profile through a Brp spot (WT, $178 \pm 2 \mathrm{~nm} ;$ rim $^{103}, 175 \pm 5 \mathrm{~nm} ; p=0.15$, Wilcoxon's rank test; Fig. 2C-E; see Materials and Methods). We detected a slight but significant ( $p=0.05$, Wilcoxon's rank test) increase in Brp ring diameter in rim mutants when computed as the diameter at halfmaximum of the line profile (WT, $325 \pm 4 \mathrm{~nm}$; rim $^{103}, 338 \pm 5$ nm; Fig. 2C-E; see Materials and Methods). Together, these data differ from the rab3 mutant but are consistent with a mutation in rab3-GAP that blocks synaptic homeostasis without major effects on anatomical neuromuscular development (Müller et al., 2011). We conclude that the defects in synaptic homeostasis in the rim mutant background are not a secondary effect attributable to altered synapse morphology, synapse development, or active zone organization assayed at the light microscopy level. The relationship between the activity of Rab3 and RIM during active zone development is examined in greater detail in a companion manuscript (Graf et al., 2012).
Evidence for decreased probability of release at the rim NMJ In rim mutants, we observe a decrease in presynaptic release without a change in the number of presynaptic active zones. This suggests that there is a decrease in the probability of presynaptic release. However, the recent demonstration of altered active zone function in rab3 mutants (Graf et al., 2009) prompted us to pursue the change in release probability more directly. To do so, we first recorded from the NMJ under two-electrode voltage clamp and confirmed a decrease in EPSC amplitude in response to single presynaptic stimuli compared with WT (Fig. $3 A-C$ ). The relative decrease in EPSC amplitude in rim mutants persisted at elevated $\left[\mathrm{Ca}^{2+}\right]_{\mathrm{e}}$, indicating that an increase in $\mathrm{Ca}^{2+}$ cannot restore the defect in release in response to single AP stimulation (Fig. 3D). Then, we stimulated the presynaptic motoneurons at $60 \mathrm{~Hz}$ and quantified the change in EPSC over time (Fig. 3A,B). WT NMJs show modest synaptic facilitation over the first 10 stimuli, whereas rim mutant NMJs display very strong facilitation ( $\sim 2.5$-fold; Fig. $3 C$, bottom). These data are consistent with a decrease in presynaptic release probability in the rim mutant and are similar to the RIM1/2 double knock-out mouse phenotype (Han et al., 2011; Kaeser et al., 2011a). Interestingly, during prolonged stimulation at $60 \mathrm{~Hz}$, rim mutant NMJs are able to achieve EPSC amplitudes that are similar in amplitude to the WT baseline EPSC evoked by single APs. This indicates that rim mutant NMJs have the capacity to release large numbers of synaptic vesicles during a stimulus train, provided that intracellular calcium is sufficiently elevated. This is consistent with a normal number of active zones (quantified above), each with a decreased number of presynaptic calcium channels. This conclusion is supported by calcium imaging experiments (see next section) and by imaging cac-GFP that is overexpressed in the rim mutant background (Graf et al., 2012).

\section{Homeostatic modulation of presynaptic calcium influx persists in rim mutants}

We next addressed the nature of the defect in the homeostatic control of transmitter release caused by loss of rim. Both genetic and imaging data demonstrate the importance of the presynaptic $\mathrm{Ca}_{\mathrm{V}} 2.1$ calcium channel in homeostatic plasticity. Point mutations in the $\mathrm{Ca}_{\mathrm{V}} 2.1$ calcium channel gene block homeostatic plasticity (Frank et al., 2006). More recently, we have demonstrated 

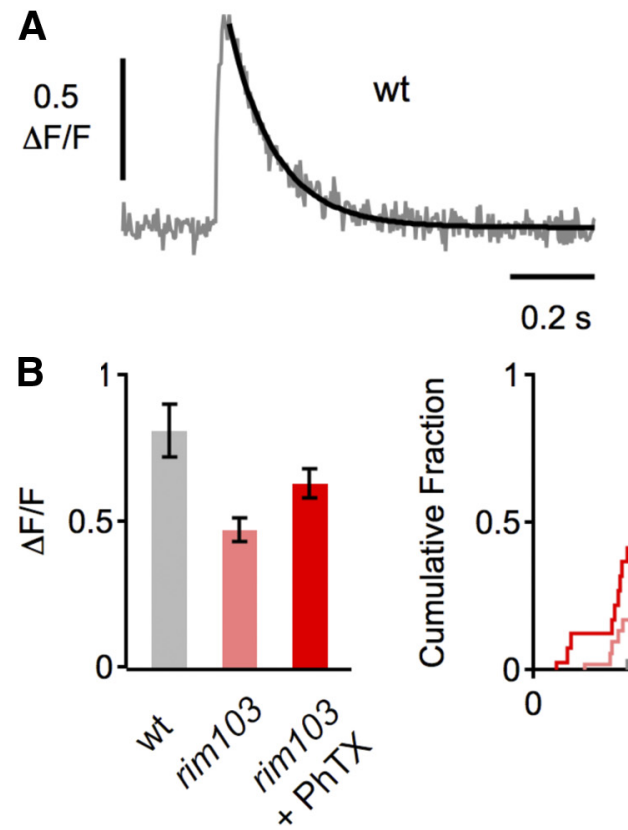
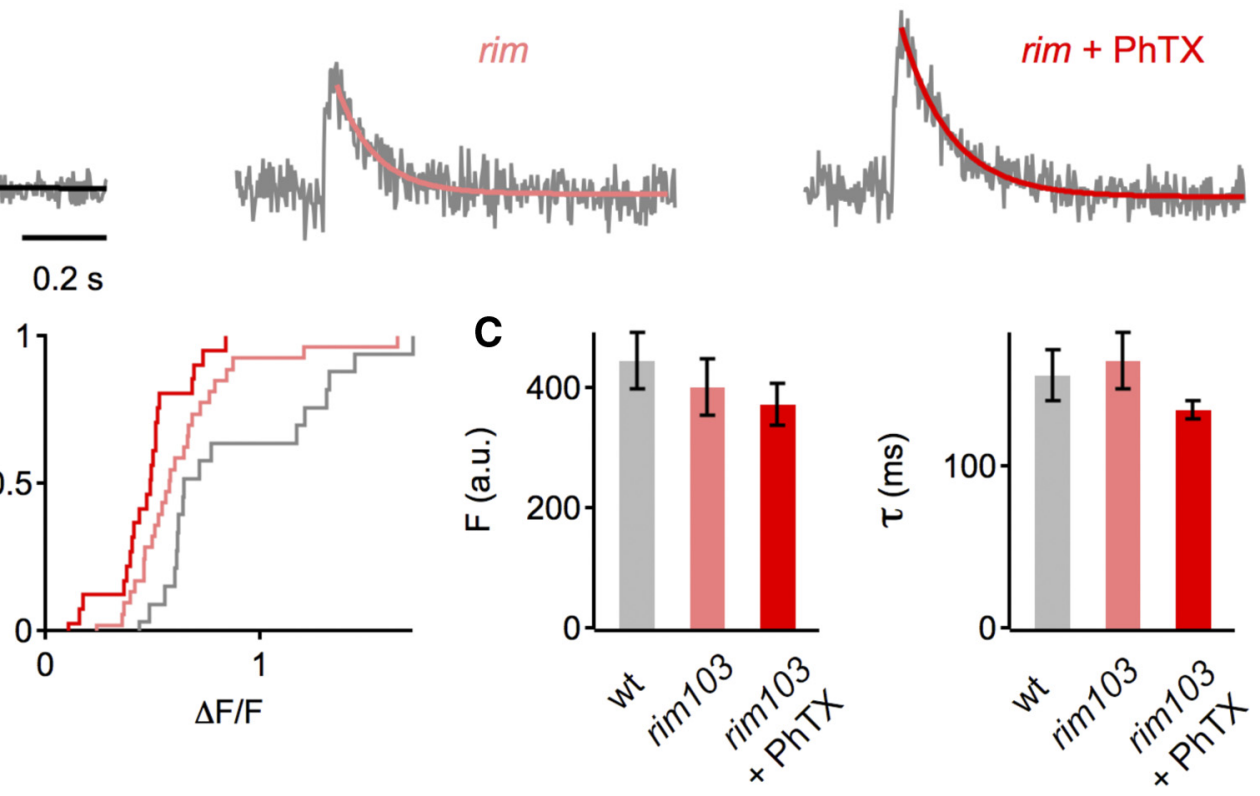

Figure 4. Relative increase in presynaptic $\mathrm{Ca}^{2+}$ influx in rim mutants after acute perturbation of glutamate receptor function. $\boldsymbol{A}$, Representative traces of spatially averaged $\mathrm{Ca}{ }^{2+}$ transients of the indicated genotypes (average of $8-12$ scans each). $\boldsymbol{B}$, Average peak amplitude of $\left(\mathrm{a}^{2+}{ }^{+}\right.$transients $(\Delta F / F)$ of control ( $n=25$ boutons; gray), rim ${ }^{103}$ without PhTX ( $n=28$; light red), and rim ${ }^{103}$ with PhTX ( $n=27$; dark red). $\mathrm{Ca}^{2+}$ transients in $\mathrm{rim}^{103}$ mutants are significantly smaller than in control under baseline conditions $(p<0.001)$, and there is a significant difference in peak amplitude between rim $^{103}$ in the absence and presence of PhTX $(p=0.02)$. C, Average baseline fluorescence $\left(F_{\text {baseline }}\right)$ and decay time constant $(\tau)$.

that homeostatic plasticity is correlated with a $30 \%$ increase in presynaptic calcium influx, a change that is sufficient to account for the near doubling of presynaptic release. Furthermore, the homeostatic modulation of presynaptic calcium influx is an essential mechanism because a point mutation in $\mathrm{Ca}_{\mathrm{V}} 2.1$ that disrupts homeostatic plasticity also blocks the homeostatic modulation of presynaptic calcium influx (Müller and Davis, 2012). RIM has been shown to interact with presynaptic voltage-gated $\mathrm{Ca}_{\mathrm{V}} 2.1$ channels in mammals (Kiyonaka et al., 2007; Kaeser et al., 2011a) and is required for normal calcium channel density in both mammals and at the Drosophila NMJ (Han et al., 2011; Kaeser et al., 2011a; Graf et al., 2012). Therefore, we asked whether loss of RIM function affects the homeostatic modulation of presynaptic $\mathrm{Ca}^{2+}$ influx.

We loaded presynaptic terminals with the $\mathrm{Ca}^{2+}$ indicator OGB-1 and imaged presynaptic spatially averaged $\mathrm{Ca}^{2+}$ transients across single boutons in response to single AP stimulation (see Materials and Methods). Under baseline conditions, we find an $\sim 42 \%$ decrease in the peak amplitude of presynaptic $\mathrm{Ca}^{2+}$ transients in rim mutants compared with WT controls $(p<$ 0.001; Fig. $4 A, B)$. This is consistent with findings at vertebrate synapses lacking RIM1/2 and likely contributes to the defect in baseline transmission of rim mutants. Interestingly, PhTXmediated induction of retrograde homeostatic signaling lead to a significant increase in the peak amplitude of presynaptic $\mathrm{Ca}^{2+}$ transients in rim mutants $(\Delta F / F=0.63 \pm 0.05)$ with respect to with control $(p<0.001)$.
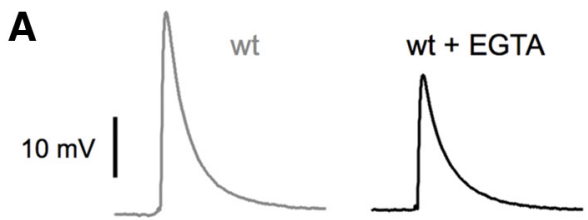

rim

$r i m+$ EGTA
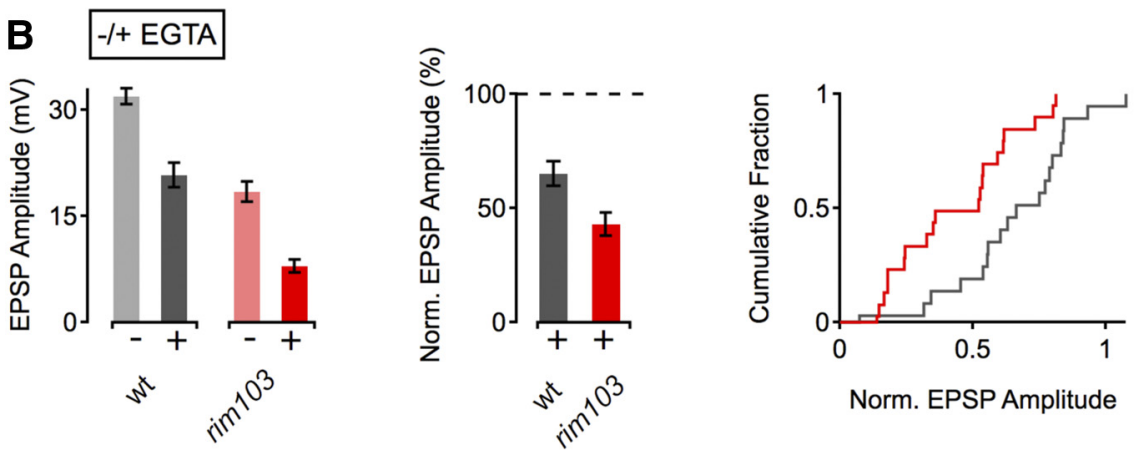

Figure 5. Increased EGTA sensitivity in rim mutants. $\boldsymbol{A}$, Example EPSP traces of WT and rim ${ }^{103}$ mutants under control conditions and after incubation in $25 \mu \mathrm{m}$ EGTA-AM for $10 \mathrm{~min}$. $\boldsymbol{B}$, Average EPSP amplitudes (left) and normalized EPSP amplitudes (middle and right) in the absence $(-)$ and presence $(+)$ of EGTA. WT (-EGTA), $n=19 ;$ WT $\left(+\right.$ EGTA), $n=19 ;$ rim $^{103}(-$ EGTA), $n=18$; $\mathrm{rim}^{103}(+\mathrm{EGTA}), n=20$. EGTA application induces a more pronounced decrease in EPSP amplitude in rim ${ }^{103}$ mutants compared

control $(\Delta F / F=0.47 \pm 0.04 ; p=0.02$; Fig. $4 A, B)$. Baseline OGB-1 fluorescence before stimulus onset and decay kinetics did not differ between the different conditions (all $p>0.05$; Fig. $4 C$ ), indicating that the difference in $\mathrm{Ca}^{2+}$ transient amplitude was not attributable to a difference in $\mathrm{Ca}^{2+}$-indicator loading, $\mathrm{Ca}^{2+}$ buffering, and/or $\mathrm{Ca}^{2+}$ extrusion. This finding demonstrates that the block of homeostatic plasticity in rim mutants is not caused by a defect in the homeostatic increase in $\mathrm{Ca}^{2+}$ influx. We conclude that RIM is necessary for normal baseline calcium influx, consistent with a defect in calcium channel density (Han et al., 2011; Kaeser et al., 2011a). How- 
A

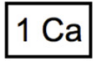

$-/+\operatorname{PhTX}$

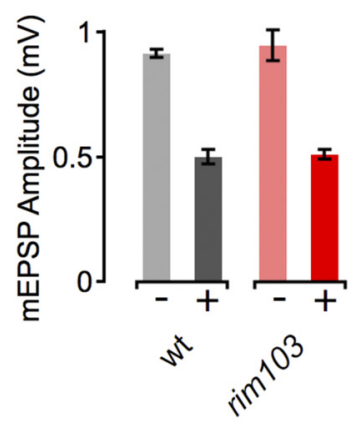

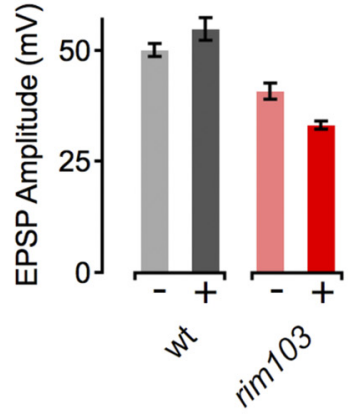
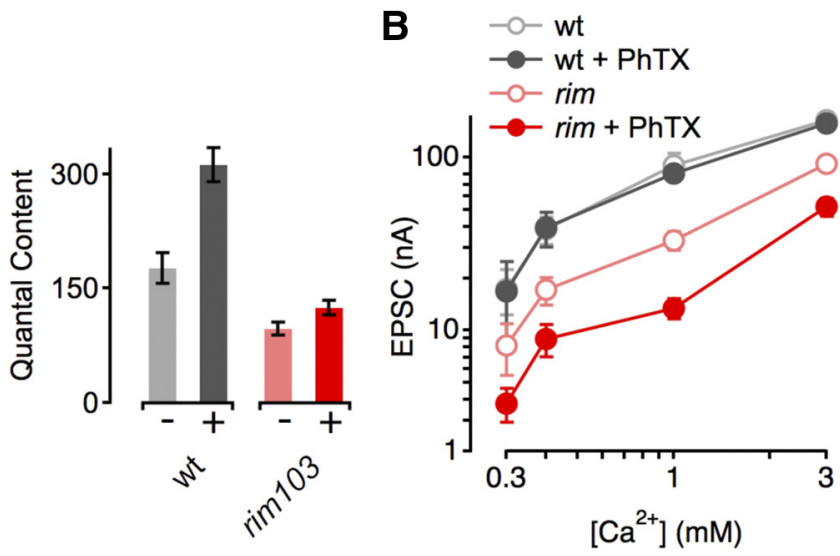

Figure 6. Increased $\left[\mathrm{Ca}^{2+}\right]_{\mathrm{e}}$ does not restore the defect in homeostatic plasticity of rim mutants. A, Average data for mEPSP amplitude (left), EPSP amplitude (middle), and quantal content (right) for indicated genotypes minus PhTX (light gray/red) and plus PhTX (black, dark red) at an $\left[\mathrm{Ca}^{2+}\right]_{\mathrm{e}}$ of $1 \mathrm{~mm} . w^{1118}(-\mathrm{PhTX}), n=10 ; w^{1118}(+\mathrm{PhTX}), n=8 ; r^{103}{ }^{103}$ $(-\mathrm{PhTX}), n=14 ;$ rim $^{103 \mathrm{c}}(+\mathrm{PhTX}), n=13$. Although PhTX application lead to a pronounced increase in quantal content in WT ( $\sim 76 \%$ of control), PhTX challenge produced a rather modest increase in quantal content ( $28 \%$ of control) in rim $^{103}$ mutants. For averages corresponding to the data presented in this figure, see Table 1. B, Relationship between mean EPSC amplitude and $\left[\mathrm{Ca}^{2+}\right]_{\mathrm{e}}$ in WT (control: light gray, $n=4-8$ per $\left[\mathrm{Ca}^{2+}\right]_{\mathrm{e}^{\prime}}$ PhTX: dark gray, $n=4-8$ per $\left[\mathrm{Ca}^{2+}\right]_{\mathrm{e}}$ ), and rim ${ }^{103}$ mutants (control: light red, $n=8-17$ per $\left[\mathrm{Ca}^{2+}\right]_{\mathrm{e}^{\prime}}$ PhTX: dark red, $n=8-14$ per $\left.\left[\mathrm{Ca}^{2+}\right]_{\mathrm{e}}\right)$. The baseline data for WT and $\mathrm{rim}^{103}$ is also shown in Figure 3D. Together, these data indicate that an increase in $\left[\mathrm{Ca}^{2+}\right]_{\mathrm{e}}$ cannot restore the defect in homeostatic plasticity seen in rim mutants.

ever, RIM is dispensable for the homeostatic modulation of presynaptic calcium influx. Indeed, it is remarkable that we observe a $24 \%$ increase in presynaptic calcium influx without a corresponding, homeostatic, change in vesicle release. These data demonstrate that a change in calcium influx is only one part of the mechanism that ultimately achieves homeostatic plasticity. Furthermore, our data imply that RIM must contribute an additional activity, downstream or in parallel to calcium influx that is essential for the homeostatic enhancement of vesicle fusion.

\section{Increased EGTA sensitivity of rim mutant synapses}

In addition to the role of RIM in $\mathrm{Ca}^{2+}$ channel clustering, RIM has been implicated in vesicle priming/docking (Weimer et al., 2006; Deng et al., 2011). To test whether rim mutants have a defect downstream of $\mathrm{Ca}^{2+}$ influx, we first probed the effects of the $\mathrm{Ca}^{2+}$ buffer EGTA on release. Because of the relatively slow $\mathrm{Ca}^{2+}$-binding rate of EGTA (Smith et al., 1984), a higher sensitivity to EGTA would be expected if the distance between $\mathrm{Ca}^{2+}$ channels and vesicles is increased. As shown in Figure 5, $A$ and $B$, EGTA-AM (25 $\mu \mathrm{M}$ for $10 \mathrm{~min}$ ) reduced WT EPSP amplitudes to $65 \%$ of control. In contrast, EGTA-AM induced a more pronounced relative decrease in EPSP amplitude in rim mutants (43\% of control; $p<0.001)$. Thus, rim mutants display an increased sensitivity of release to EGTA. This indicates that the average distance between the sites of $\mathrm{Ca}^{2+}$ influx and synaptic vesicles is larger in rim mutants, consistent with observations at mammalian central synapses (Han et al., 2011; Kaeser et al., 2011a).

To test whether the increased distance between $\mathrm{Ca}^{2+}$ channels and vesicles might impair homeostatic plasticity in rim mutants, we probed synaptic homeostasis in rim mutants at an increased $\left[\mathrm{Ca}^{2+}\right]_{\mathrm{e}}(1 \mathrm{~mm}$; Fig. $6 \mathrm{~A})$. The resulting larger intra-terminal $\mathrm{Ca}^{2+}$ microdomains might be expected to supersede a defect in the "coupling" between $\mathrm{Ca}^{2+}$ channels and vesicles and thereby restore homeostatic plasticity in rim mutants. However, although increasing $\left[\mathrm{Ca}^{2+}\right]_{\mathrm{e}}$ to $1 \mathrm{~mm}$ caused a pronounced increase in baseline transmission, it created a very modest (28\%) restoration of homeostatic plasticity, an effect that remains dramatically less than the $76 \%$ increase in release observed in WT animals (Fig.
$6 A)$. We next assayed synaptic homeostasis at four different $\left[\mathrm{Ca}^{2+}\right]_{\mathrm{e}}$ using two-electrode voltage clamp (0.3-3 mM; Fig. 6B). Although there was no significant difference in EPSC amplitude in WT in the presence or absence of PhTX (Fig. 6B, gray data; $p>0.59$ at all $\left[\mathrm{Ca}^{2+}\right]_{\mathrm{e}}$ ), PhTX treatment lead to a significant reduction of EPSC amplitude in rim $^{103}$ mutants at most $\mathrm{Ca}^{2+}$ concentrations tested (Fig. $6 B$, red data; $p<0.03$ for $0.4-3 \mathrm{~mm}\left[\mathrm{Ca}^{2+}\right]_{\mathrm{e}} ; p=0.07$ at $\left.0.3 \mathrm{~mm}\left[\mathrm{Ca}^{2+}\right]_{\mathrm{e}}\right)$. Thus, the defect in homeostasis persists at elevated $\left[\mathrm{Ca}^{2+}\right]_{\mathrm{e}}$. Based on these data, we propose that an increase in the average distance between $\mathrm{Ca}^{2+}$ channels and vesicles is unlikely to be the primary underlying defect that prevents homeostatic plasticity in rim mutants (see Discussion).

\section{RIM-dependent modulation of the RRP is required for the expression of synaptic homeostasis}

Recently, it has been shown that the homeostatic enhancement of presynaptic release at the Drosophila NMJ is correlated with an increase in the apparent size of the RRP (Weyhersmüller et al., 2011). However, to date, there is no molecular or genetic manipulation that specifically disrupts the modulation of the RRP, and, therefore, it is unknown whether a change in the RRP is required for homeostatic plasticity.

We therefore first probed RRP size by cumulative EPSC amplitude analysis during stimulus trains at elevated $\left[\mathrm{Ca}^{2+}\right]_{\mathrm{e}}(3 \mathrm{mM}$; Fig. 7A-C; Schneggenburger et al., 1999). We verified that a homeostatic enhancement of release is correlated with an increase in RRP size, as indicated by a significant increase in the number of readily-releasable vesicles at WT synapses during PhTX application (Fig. 7C, right; $p=0.031$; Weyhersmüller et al., 2011). Next, we reveal a significant decrease in the RRP size in rim mutants compared with WT controls under baseline conditions (Fig. $7 A-C ; p=0.028$ ), similar to findings in RM1/2 double knock-out mice (Deng et al., 2011; Han et al., 2011; Kaeser et al., 2011a). Then we assayed the RRP after application of PhTX to the rim mutant and found that there was no significant increase in vesicle pool size in rim mutants with respect to baseline (Fig. $7 C$, right; $p=0.46$ ). These findings were confirmed by an independent set of experiments at the lower standard $\left[\mathrm{Ca}^{2+}\right]_{\mathrm{e}}$ of this study $(0.4$ $\mathrm{mM}$; Fig. 7D). In good agreement with the data collected at in- 
creased $\mathrm{Ca}^{2+}$ concentration (Fig. $7 A-C$ ), baseline RRP size of rim mutant synapses was significantly smaller than in WT controls $(p=0.008$; Fig. $7 D$, right; note that the RRP size estimate at low $\left[\mathrm{Ca}^{2+}\right]_{\mathrm{e}}$ is based on a later steady-state region; see Materials and Methods), and PhTX application did not induce a significant increase in RRP size in rim mutants with respect to baseline (Fig. $7 D$, right; $p=$ $0.6)$. These data suggest that RIM is necessary for the homeostatic increase in RRP size.

To further support this conclusion, we used EPSC amplitude fluctuation analysis to estimate the number of release-ready vesicles (Fig. 8; see Materials and Methods). PhTX application reduced quantal size $(q)$ with respect to baseline at both WT $(p=0.05)$ and rim mutant $(p=$ 0.001 ; Fig. $8 D$, left) synapses. At WT synapses, we observed a significant increase in the binomial parameter $N$ after PhTX treatment, indicating an increase in RRP size ( $p=0.039$; Fig. $8 D$, right; Weyhersmüller et al., 2011). In contrast, there was no significant difference in $N$ between PhTX-treated rim NMJs and baseline controls ( $p=0.2$; Fig. $8 D$, right). Together, data from three different sets of experiments and two different approaches indicate that the rim mutation blocks the relative increase in RRP size that is normally correlated with a homeostatic potentiation of presynaptic release (Weyhersmüller et al., 2011). We conclude that rim mutations block homeostatic plasticity by preventing the modulation of the RRP downstream of change in presynaptic calcium influx. By extension, homeostatic plasticity appears to require two genetically separable processes, an enhancement of presynaptic calcium influx (Müller and Davis, 2012) and a modulation of the RRP.

\section{Genetic interaction between rim and} rab3-GAP during synaptic homeostasis If RIM is an essential component of the presynaptic machinery that effects a homeostatic enhancement of presynaptic neurotransmitter release, then we predict that there will be genetic interactions between RIM and other previously identified homeostatic signaling molecules, including Rab3-GAP. Because both rab3-GAP and rim mutations alone are sufficient to completely block synaptic homeostasis, analysis of the double mutant would not be informative. However, we are able to test whether synaptic homeostasis is blocked in the double heterozygous mutant combination, as performed previously (Dickman and Davis, 2009; Frank et al., 2009). Synaptic homeostasis is normal in heterozygous mutations in either rab3-GAP (rab3-GAP/+) or rim $\left(\mathrm{rim}^{\mathrm{MB}} /+\right.$ ) (Fig. 9A; Müller et al., 2011). However, in the double heterozygous rab3GAP/+; rim $^{M B} /+$ mutants, PhTX incubation did not induce significant homeostatic compensation ( $p=0.17$; Fig. $8 A$, bottom).
B wt rim103

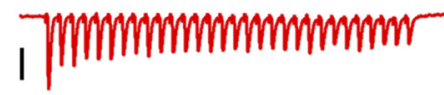
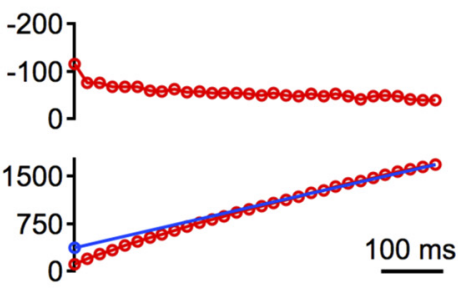

$100 \mathrm{~ms}$

$00 \mathrm{~ms}$
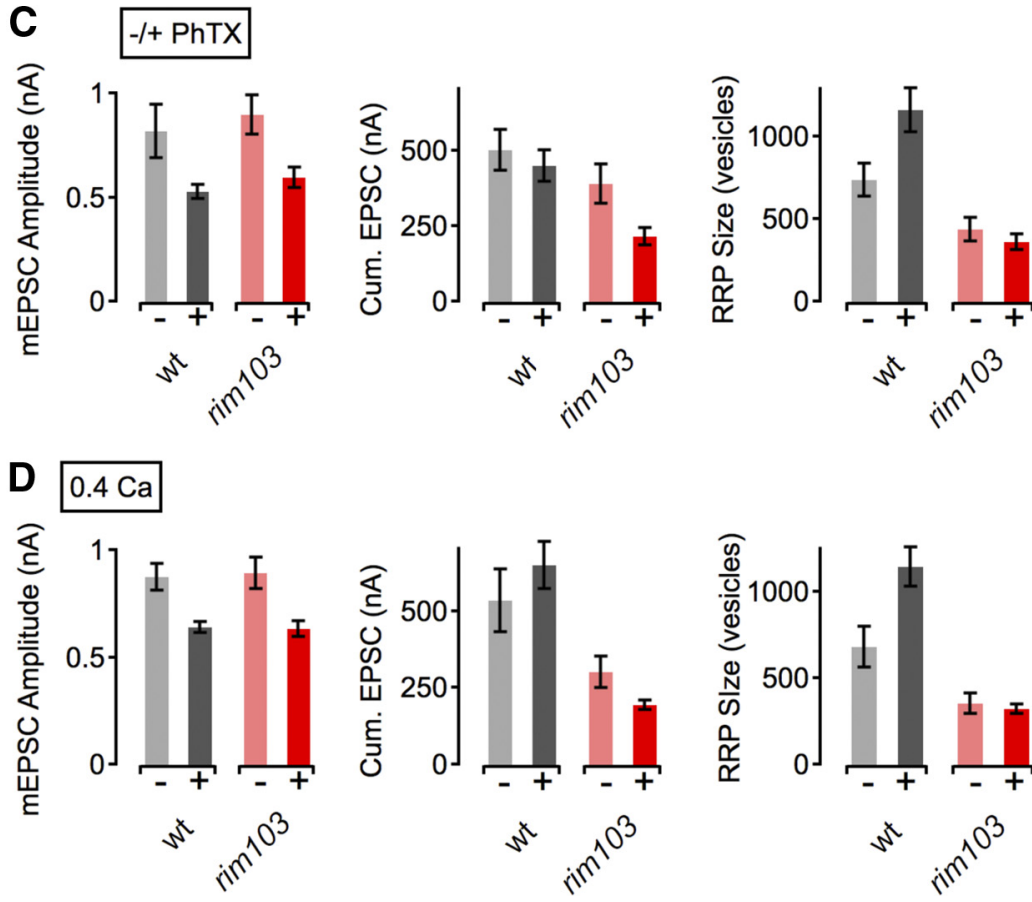

Figure 7. RIM-dependent modulation of the RRP is required for synaptic homeostasis. $A$, Example EPSC trace (top), peak EPSC amplitudes (middle), and cumulative EPSC amplitudes (Cum. EPSC, bottom) evoked by 60-Hz stimulation (30 stimuli) of a WT NMJ at $3 \mathrm{~mm}$ Example data for a rim $^{103}$ synapse, same as in A. C, Average mEPSC amplitude (left), cumulative EPSC amplitude (Cum. EPSC, middle), and RRP size (right) for the indicated genotypes minus PhTX (light gray/red) and plus PhTX (black, dark red) at $3 \mathrm{~mm}\left[\mathrm{Ca}^{2+}\right]_{\mathrm{e}} \cdot w^{1118}(-\mathrm{PhTX})$, $n=6 ; w^{1118}(+\mathrm{PhTX}), n=5 ; \operatorname{rim}^{103}(-\mathrm{PhTX}), n=6 ; \mathrm{rim}^{103}(+\mathrm{PhTX}), n=5 . \mathrm{D}$, Corresponding average data (asin C) at $\left.0.4 \mathrm{~mm}^{1 \mathrm{Ca}^{2+}}\right]_{\mathrm{e}^{\prime}}$ $w^{1118}(-\mathrm{PhTX}), n=13 ; w^{1118}(+\mathrm{PhTX}), n=13 ; \operatorname{rim}^{103}(-\mathrm{PhTX}), n=13 ; \operatorname{rim}^{103}(+\mathrm{PhTX}), n=13$. Although PhTX application led to $\left.\left[\mathrm{Ca}^{2+}\right]_{\mathrm{e}^{\prime}} p=0.02\right)$, there was no significant increase in vesicle pool size in rim mutants $\left(\boldsymbol{C}, \boldsymbol{D}\right.$, right, red data; $3 \mathrm{~mm}\left[\mathrm{Ca}^{2+}\right]_{\mathrm{e}^{\prime}} p=0.46 ; 0.4$ $\left.\mathrm{mm}\left[\mathrm{Ca}^{2+}\right]_{\mathrm{e}^{\prime}} p=0.6\right)$. For averages corresponding to the data presented in this figure, see Table 1.

This result is important for two reasons. First, these data are consistent with previous biochemical experiments suggesting that RIM should function in concert with Rab3/Rab3-GAP. Second, these data provide additional confirmation that RIM is necessary for synaptic homeostasis because a heterozygous mutation is sufficient to cause a block of synaptic homeostasis when combined with a mutation of a previously characterized homeostatic signaling molecule.

\section{Discussion}

Here we demonstrate that RIM is required for the retrograde, homeostatic enhancement of presynaptic neurotransmitter at the Drosophila NMJ. Three independent mutations in the RIM gene, including a small internal deletion, block the homeostatic increase of 
A

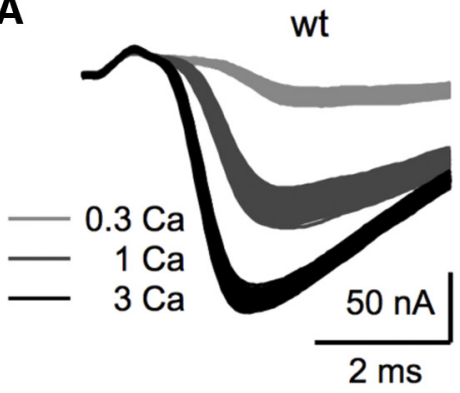

$\operatorname{rim} 103$

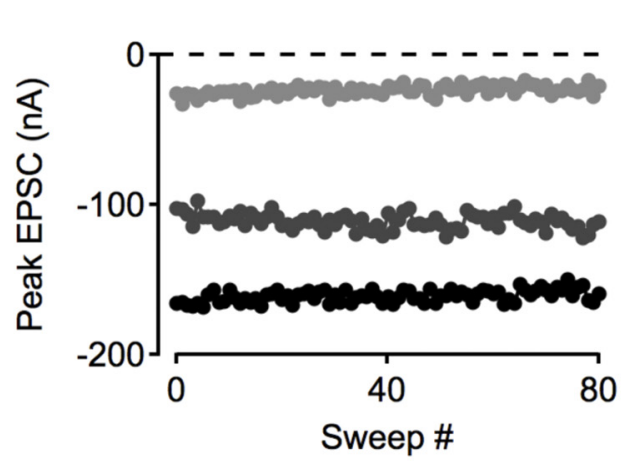

D

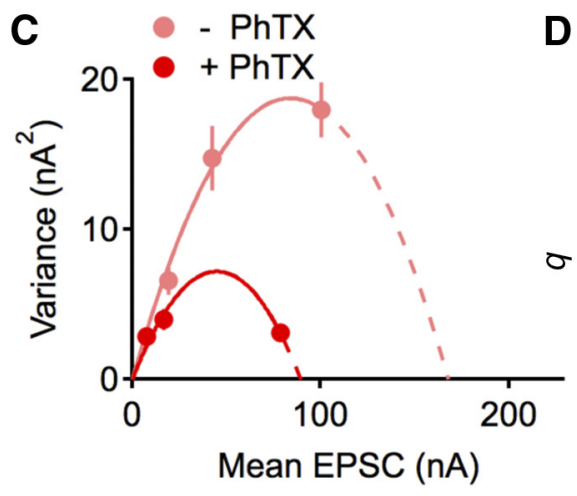

$-1+\mathrm{PhTX}$

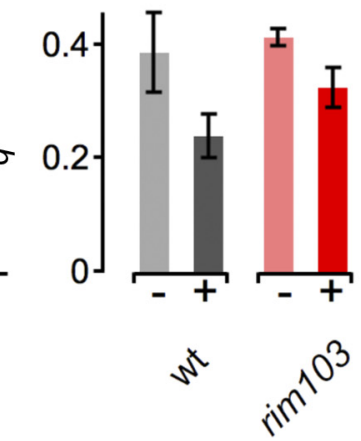

B

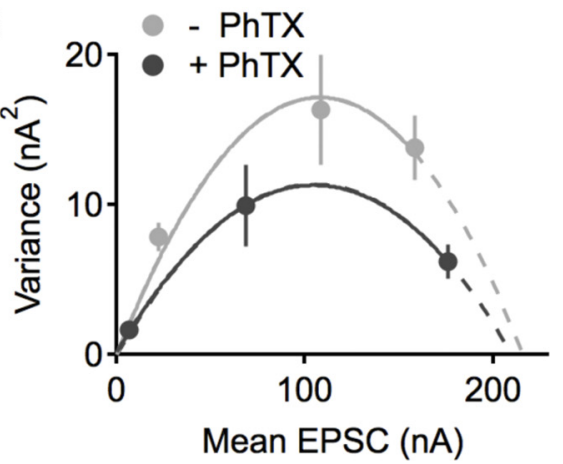

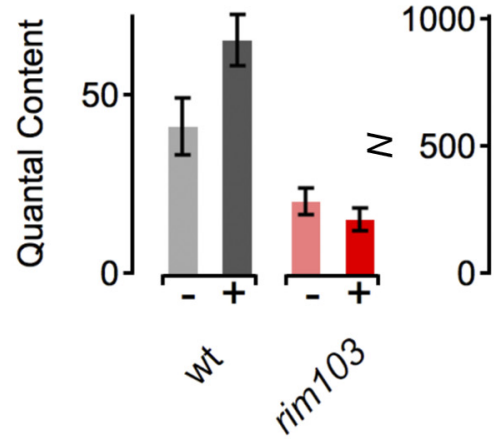

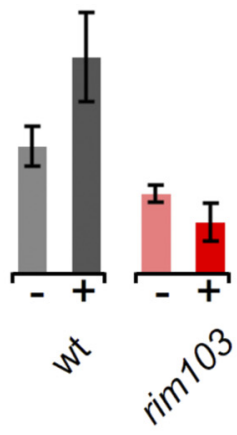

Figure 8. Variance-mean analysis supports a RIM-dependent modulation of the RRP during synaptic homeostasis. $A$, Example EPSC traces (left) and EPSC peak amplitudes as a function of sweep number of a WT NMJ at the indicated $\left[\mathrm{Ca}^{2+}\right]_{\mathrm{e}}$ (in millimolar). $\boldsymbol{B}$, Example EPSC amplitude variance-mean plots of two WT synapses in the absence (gray) and presence (black) of PhTX with parabola fits (solid lines) that were extrapolated to the $x$-intercept (dashed lines). C, Example variance-mean plots of two rim ${ }^{103} \mathrm{NMJ}$ s in the absence (light red) and presence (dark red) of PhTX. D, Average data for the quantal parameter $q$ (quantal size, left), quantal content at $1 \mathrm{~mm}\left[\mathrm{Ca}^{2+}\right]_{\mathrm{e}}$ (middle), and the binomial parameter $N$ (number of release sites, right). $w^{1118}(-\mathrm{PhTX}), n=4 ; w^{1118}$ $\left.(+\mathrm{PhTX}), n=4 ; r_{i m}^{103}(-\mathrm{PhTX}), n=9 ; r_{i m}^{103}(+\mathrm{PhTX}), n=8\right)$. Note that PhTX application induced an increase in the binomial parameter Nin WT (gray/black, right) that was blocked by rim ${ }^{103}$ (light red/dark red, right).

presynaptic release (Fig. 1). In addition, neuronal expression of UAS-rim RNAi blocks synaptic homeostasis (Fig. 1). The defect in homeostatic potentiation does not appear to result from major changes in synapse or active zone morphology (Fig. 2). Furthermore, the defect in homeostatic potentiation is observed over a 10 -fold range of $\left[\mathrm{Ca}^{2+}\right]_{\mathrm{e}}(0.3-3 \mathrm{~mm}$; Fig. 6). We then present evidence that RIM has two independent functions, only one of which is required for homeostatic plasticity. First, we provide evidence that RIM is necessary for normal presynaptic calcium influx and presynaptic release probability (Figs. 3, 4). This is consistent with a wellestablished role for RIM in binding to presynaptic calcium channels (Kiyonaka et al., 2007; Kaeser et al., 2011a) and concentrating calcium channels to the active zone (Han et al., 2011; Kaeser et al., 2011a). However, the regulation of presynaptic calcium channel density does not appear to be the function that RIM contributes to the mechanisms of synaptic homeostasis. The homeostatic modulation of presynaptic calcium influx is still observed in the rim mutant, implying that the homeostatic, retrograde signaling system remains functional and is able to modulate calcium channel number or function in the absence of rim.

We provide evidence that the required function of RIM during homeostatic plasticity is the modulation of the RRP. We have confirmed that synaptic homeostasis is correlated with an increase in the RRP. We then demonstrate that the homeostatic increase in the RRP is blocked in the rim mutation (Figs. 7, 8). This observation is significant for several reasons. First, these data provide evidence that a change in the RRP is not just correlated with homeostatic plasticity but is required. Second, these data imply that the homeostatic mod- ulation of RRP size can be controlled independently of, or in parallel with, the homeostatic modulation of presynaptic calcium influx that persists in the rim mutant. Third, our data provide evidence that RIM imparts an essential activity that is required for the homeostatic modulation of the RRP during homeostatic plasticity. Finally, our data indicate that the precise homeostatic modulation of presynaptic neurotransmitter release occurs at the intersection of two independently regulated processes, calcium influx and the RRP size. This suggests new complexity underlying the expression of homeostatic plasticity within the presynaptic nerve terminal.

\section{Precise modulation of presynaptic neurotransmitter release during synaptic homeostasis}

One of the remarkable features of homeostatic plasticity is that it is a quantitatively accurate form of synaptic modulation. It has been shown that the change in presynaptic neurotransmitter release precisely offsets the change in postsynaptic glutamate receptor function. More specifically, a $20 \%$ decrease in receptor sensitivity is offset by a $20 \%$ increase in presynaptic release, whereas a $50 \%$ decrease in receptor sensitivity is offset by a $50 \%$ increase in release (Frank et al., 2006). This is precisely what is expected of a true homeostatic signaling system that restores a system to baseline, or set-point functionality. However, this capacity of a homeostatic signaling system also places an unusual demand on the signaling system controlling the modulation presynaptic neurotransmitter release. It makes intuitive sense that a change in presynaptic calcium influx is involved in homeostatic plasticity (Frank et al., 2006, 2009; Müller and Davis, 2012). 
However, the highly cooperative nature of calcium-dependent vesicle fusion would necessitate that calcium influx be controlled very precisely during homeostatic plasticity to prevent overshoot and inappropriate potentiation of vesicle release. Therefore, it might also make sense for the expression of synaptic homeostasis to require a parallel change within the presynaptic nerve terminal, in addition to a modulation of presynaptic calcium influx. Our data suggest that the RIMdependent homeostatic modulation of RRP size is one such mechanism that is independent of calcium influx and necessary, in parallel, for the expression of homeostatic plasticity (Fig. 9B). If both mechanisms can limit the amount of vesicle release, this would provide an additional opportunity to control or limit the extent of homeostatic plasticity.

\section{RIM-dependent modulation of RRP size}

How might RIM participate in the regulated modulation of RRP size? First, synaptic homeostasis at the Drosophila NMJ requires a retrograde signal from muscle to nerve (Frank et al., 2006). We hypothesized previously that this retrograde signal is able to directly influence presynaptic calcium channel number or function (Frank et al., 2006, 2009), and genetic evidence has been provided that Eph-Ephexin signaling could impinge on the regulation of presynaptic $\mathrm{Ca}_{\mathrm{V}} 2.1$ calcium channels (Frank et al., 2009). However, it remains unknown whether EphEphexin signaling functions during the rapid induction of homeostatic plasticity and whether this signaling system participates in the modulation of the RRP. It is formally possible that an independent retrograde signal targets RIM and the regulation of the RRP.

In mammalian systems, RIM has been shown to interact with numerous proteins, including Rab3 and the presynaptic calcium channel. The zinc finger domain of RIM has also been shown to interact with Munc13 and has been implicated in the vesicle priming/docking function of RIM. Rab3-GAP and Rab3-dependent signaling have been implicated in synaptic homeostasis (Müller et al., 2011). It is currently unknown how Rab3 signaling might intersect with RIM to participate in homeostatic plasticity, and this is something that could be addressed in the future. However, it is interesting to speculate that RIM-dependent regulation of vesicle docking/ priming via Munc13 could be a function of RIM that is required for the homeostatic modulation of RRP size during homeostatic plasticity. Mutations in the Drosophila homolog of Munc13 are late embryonic lethal, preventing a straightforward analysis during homeostatic plasticity. This could be addressed in the future through protein knockdown and a molecular dissection of the RIM-Dunc13 protein interaction.

RIM binding protein (RBP) function was analyzed recently at the Drosophila NMJ (Liu et al., 2011). RBP mutations cause a
B Baseline

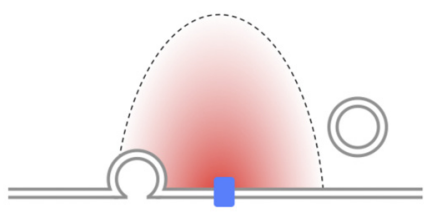

Homeostatic plasticity

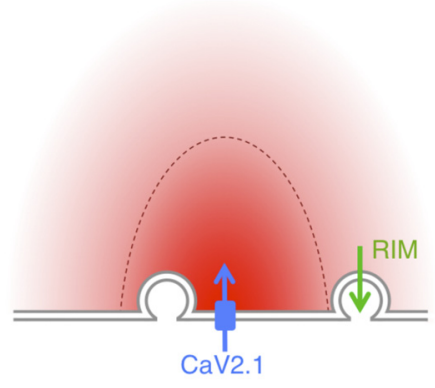

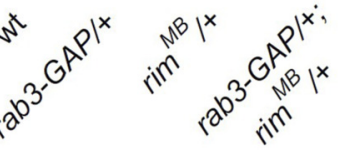

Figure 9. rim interacts genetically with rab3-GAP during synaptic homeostasis. A, Average data for mEPSP amplitude (top), +PhTX), $n=11 ;$ rab3-GAPI+ (-PhTX), $n=13 ;$ rab3-GAP/ + (+PhTX), $n=15 ; r^{M B B} /+(-\mathrm{PhTX}), n=12 ; r^{\mathrm{MB}} /+$ +PhTX), $n=12 ; \mathrm{rab3}-G A P /$ rim $^{\mathrm{MB}}(-\mathrm{PhTX}), n=18 ; \mathrm{rab3}_{-}-\mathrm{GAP} /$ rim $^{\mathrm{MB}}(+\mathrm{PhTX}), n=20$. Homeostatic compensation is norma (red) and rab3-GAP/+ heterozygous mutations (blue). Homeostasis is blocked when these two heterozygous ations are combined ( rim $^{M B} /+;$ rab3-GAP/+, magenta) (quantal content, $-1+\mathrm{PhTX}^{\prime} p=0.17$ ). All data represent mean SEM. B, Emerging model for rim function during synaptic homeostasis. Top, Under baseline conditions, $\mathrm{Ca}^{2+}$ influx through domain (red). Vesicles that are located outside the $\mathrm{Ca}^{2+}$ domain and/or that are not docked/primed (right vesicle at a distance from the membrane) are not released. Bottom, 0ur data suggest that two processes are required for the expression of homeostatic plasticity. First, there is an increase in $\mathrm{Ca}^{2+}$ influx through $\mathrm{Ca}_{\mathrm{v}} 2.1$ channels (blue arrow). Second, there is a RIM-dependent docking/priming step (green arrow) that leads to an increase in the RRP. Disruption of either of these two processes alone is sufficient to prevent the expression of homeostatic plasticity.

deficit in calcium influx and calcium channel abundance that are quantitatively similar to the changes observed here in rim mutants. Although RBP mutations show a more severe defect in baseline transmission in response to a single AP, the RBP mutant synapse shows dramatic facilitation similar to that observed in rim mutants, indicating that the synapse is capable of substantial vesicle release provided sufficient presynaptic calcium entry during a stimulus train. Because the defect in calcium influx is similar when comparing rim and RBP mutants, it seems that additional deficits contribute to the $R B P$ release phenotype, which could include the observed active zone disorganization (Liu et al., 2011). It remains unknown whether RBP is required for homeostatic synaptic plasticity, but the existing data are consistent with these two proteins functioning in concert to control active zone function and, potentially, homeostatic synaptic plasticity.

\section{References}

Bergquist S, Dickman DK, Davis GW (2010) A hierarchy of cell intrinsic and target-derived homeostatic signaling. Neuron 66:220-234. CrossRef Medline

Brown TH, Perkel DH, Feldman MW (1976) Evoked neurotransmitter re- 
lease: statistical effects of nonuniformity and nonstationarity. Proc Natl Acad Sci U S A 73:2913-2917. CrossRef Medline

Calakos N, Schoch S, Südhof TC, Malenka RC (2004) Multiple roles for the active zone protein RIM1alpha in late stages of neurotransmitter release. Neuron 42:889-896. CrossRef Medline

Castillo PE, Schoch S, Schmitz F, Südhof TC, Malenka RC (2002) RIMlalpha is required for presynaptic long-term potentiation. Nature 415:327-330. CrossRef Medline

Castillo PE, Chiu CQ, Carroll RC (2011) Long-term plasticity at inhibitory synapses. Curr Opin Neurobiol 21:328-338. CrossRef Medline

Davis GW (2006) Homeostatic control of neural activity: from phenomenology to molecular design. Annu Rev Neurosci 29:307-323. CrossRef Medline

Davis GW, Goodman CS (1998) Synapse-specific control of synaptic efficacy at the terminals of a single neuron. Nature 392:82-86. CrossRef Medline

Deng L, Kaeser PS, Xu W, Südhof TC (2011) RIM proteins activate vesicle priming by reversing autoinhibitory homodimerization of Munc13. Neuron 69:317-331. CrossRef Medline

Dickman DK, Davis GW (2009) The schizophrenia susceptibility gene dysbindin controls synaptic homeostasis. Science 326:1127-1130. CrossRef Medline

Dietzl G, Chen D, Schnorrer F, Su KC, Barinova Y, Fellner M, Gasser B, Kinsey K, Oppel S, Scheiblauer S, Couto A, Marra V, Keleman K, Dickson BJ (2007) A genome-wide transgenic RNAi library for conditional gene inactivation in Drosophila. Nature 448:151-156. CrossRef Medline

Fouquet W, Owald D, Wichmann C, Mertel S, Depner H, Dyba M, Hallermann S, Kittel RJ, Eimer S, Sigrist SJ (2009) Maturation of active zone assembly by Drosophila Bruchpilot. J Cell Biol 186:129-145. CrossRef Medline

Frank CA, Kennedy MJ, Goold CP, Marek KW, Davis GW (2006) Mechanisms underlying the rapid induction and sustained expression of synaptic homeostasis. Neuron 52:663-677. CrossRef Medline

Frank CA, Pielage J, Davis GW (2009) A presynaptic homeostatic signaling system composed of the Eph receptor, Ephexin, Cdc42, and CaV2.1 calcium channels. Neuron 61:556-569. CrossRef Medline

Graf ER, Daniels RW, Burgess RW, Schwarz TL, DiAntonio A (2009) Rab3 dynamically controls protein composition at active zones. Neuron 64: 663-677. CrossRef Medline

Graf ER, Valakh V, Wright CM, Wu C, Liu Z, Zhang YQ, DiAntonio A (2012) RIM promotes calcium channel accumulation at active zones of the Drosophila neuromuscular junction. J Neurosci 32:16586-16596.

Hallermann S, Heckmann M, Kittel RJ (2010) Mechanisms of short-term plasticity at neuromuscular active zones of Drosophila. HFSP Journal 4:72-84.

Han Y, Kaeser PS, Südhof TC, Schneggenburger R (2011) RIM determines $\mathrm{Ca}^{2+}$ channel density and vesicle docking at the presynaptic active zone. Neuron 69:304-316. CrossRef Medline

Kaeser PS, Deng L, Wang Y, Dulubova I, Liu X, Rizo J, Südhof TC (2011a) RIM proteins tether $\mathrm{Ca}^{2+}$ channels to presynaptic active zones via a direct PDZ-domain interaction. Cell 144:282-295. CrossRef Medline

Kittel RJ, Wichmann C, Rasse TM, Fouquet W, Schmidt M, Schmid A, Wagh DA, Pawlu C, Kellner RR, Willig KI, Hell SW, Buchner E, Heckmann M, Sigrist SJ (2006) Bruchpilot promotes active zone assembly, $\mathrm{Ca}^{2+}$ channel clustering, and vesicle release. Science 312:1051-1054. CrossRef Medline

Kiyonaka S, Wakamori M, Miki T, Uriu Y, Nonaka M, Bito H, Beedle AM, Mori E, Hara Y, De Waard M, Kanagawa M, Itakura M, Takahashi M, Campbell KP, Mori Y (2007) RIM1 confers sustained activity and neurotransmitter vesicle anchoring to presynaptic $\mathrm{Ca}^{2+}$ channels. Nat Neurosci 10:691-701. CrossRef Medline

Koushika SP, Richmond JE, Hadwiger G, Weimer RM, Jorgensen EM, Nonet ML (2001) A post-docking role for active zone protein Rim. Nat Neurosci 4:997-1005. CrossRef Medline

Liu KSY, Siebert M, Mertel S, Knoche E, Wegener S, Wichmann C, Matkovic T, Muhammad K, Depner H, Mettke C, Bückers J, Hell SW, Müller M, Davis GW, Schmitz D, Sigrist SJ (2011) RIM-binding protein, a central part of the active zone, is essential for neurotransmitter release. Science 334:1565-1569.

Marder E, Goaillard JM (2006) Variability, compensation and homeostasis in neuron and network function. Nat Rev Neurosci 7:563-574. CrossRef Medline

Martin AR (1955) A further study of the statistical composition on the endplate potential. J Physiol 130:114-122. Medline

Meyer AC, Neher E, Schneggenburger R (2001) Estimation of quantal size and number of functional active zones at the calyx of held synapse by nonstationary EPSC variance analysis. J Neurosci 21:7889-7900. Medline

Miśkiewicz K, Jose LE, Bento-Abreu A, Fislage M, Taes I, Kasprowicz J, Swerts J, Sigrist S, Versées W, Robberecht W, Verstreken P (2011) ELP3 controls active zone morphology by acetylating the ELKS family member Bruchpilot. Neuron 72:776-788. CrossRef Medline

Mittelstaedt T, Alvaréz-Baron E, Schoch S (2010) RIM proteins and their role in synapse function. Biol Chem 391:599-606. Medline

Müller M, Davis GW (2012) Transsynaptic control of presynaptic $\mathrm{Ca}^{2+}$ influx achieves homeostatic potentiation of neurotransmitter release. Curr Biol 22:1102-1108. CrossRef Medline

Müller M, Pym EC, Tong A, Davis GW (2011) Rab3-GAP controls the progression of synaptic homeostasis at a late stage of vesicle release. Neuron 69:749-762. CrossRef Medline

Pielage J, Cheng L, Fetter RD, Carlton PM, Sedat JW, Davis GW (2008) A presynaptic giant ankyrin stabilizes the NMJ through regulation of presynaptic microtubules and transsynaptic cell adhesion. Neuron 58 : 195-209. CrossRef Medline

Scheuss V, Neher E (2001) Estimating synaptic parameters from mean, variance, and covariance in trains of synaptic responses. Biophysical J 81:1970-1989. CrossRef Medline

Scheuss V, Schneggenburger R, Neher E (2002) Separation of presynaptic and postsynaptic contributions to depression by covariance analysis of successive EPSCs at the calyx of held synapse. J Neurosci 22:728-739. Medline

Schindelin J, Arganda-Carreras I, Frise E, Kaynig V, Longair M, Pietzsch T, Preibisch S, Rueden C, Saalfeld S, Schmid B, Tinevez J-Y, White DJ, Hartenstein V, Eliceiri K, Tomancak P, Cardona A (2012) Fiji: an opensource platform for biological-image analysis. Nat Methods 9:676-682. CrossRef Medline

Schneggenburger R, Meyer AC, Neher E (1999) Released fraction and total size of a pool of immediately available transmitter quanta at a calyx synapse. Neuron 23:399-409. CrossRef Medline

Schoch S, Castillo PE, Jo T, Mukherjee K, Geppert M, Wang Y, Schmitz F, Malenka RC, Südhof TC (2002) RIMlalpha forms a protein scaffold for regulating neurotransmitter release at the active zone. Nature 415 : 321-326. CrossRef Medline

Schoch S, Mittelstaedt T, Kaeser PS, Padgett D, Feldmann N, Chevaleyre V, Castillo PE, Hammer RE, Han W, Schmitz F, Lin W, Südhof TC (2006) Redundant functions of RIM1alpha and RIM2alpha in $\mathrm{Ca}^{2+}$-triggered neurotransmitter release. EMBO J 25:5852-5863. CrossRef Medline

Silver RA, Momiyama A, Cull-Candy SG (1998) Locus of frequency-dependent depression identified with multiple-probability fluctuation analysis at rat climbing fibre-Purkinje cell synapses. J Physiol 510:881-902. CrossRef Medline

Smith PD, Liesegang GW, Berger RL, Czerlinski G, Podolsky RJ (1984) A stopped-flow investigation of calcium ion binding by ethylene glycol bis(beta-aminoethyl ether)- $N, N^{\prime}$-tetraacetic acid. Anal Biochem 143: 188-195. CrossRef Medline

Taschenberger H, Scheuss V, Neher E (2005) Release kinetics, quantal parameters and their modulation during short-term depression at a developing synapse in the rat CNS. J Physiol 568:513-537. CrossRef Medline

Wang Y, Südhof TC (2003) Genomic definition of RIM proteins: evolutionary amplification of a family of synaptic regulatory proteins. Genomics 81:126-137. CrossRef Medline

Wang Y, Okamoto M, Schmitz F, Hofmann K, Südhof TC (1997) Rim is a putative Rab3 effector in regulating synaptic-vesicle fusion. Nature 388: 593-598. CrossRef Medline

Weimer RM, Gracheva EO, Meyrignac O, Miller KG, Richmond JE, Bessereau JL (2006) UNC-13 and UNC-10/Rim localize synaptic vesicles to specific membrane domains. J Neurosci 26:8040-8047. CrossRef Medline

Weyhersmüller A, Hallermann S, Wagner N, Eilers J (2011) Rapid active zone remodeling during synaptic plasticity. J Neurosci 31:6041-6052. CrossRef Medline 